\title{
Processability of PEEK, a new polymer for High Temperature Laser Sintering (HT-LS)
}

\author{
S. Berretta, K.E. Evans, 0. Ghita \\ University of Exeter, College of Engineering, Mathematics and Physical Sciences, EX4 4QF, \\ Exeter, United Kingdom
}

Corresponding author

Silvia Berretta: sb508@exeter.ac.uk

\begin{abstract}
Currently, the HT-LS sector is predominantly based around one commercial poly ether ketone (PEK) polymer. Although the combination of polymer and process works well, a lower melting temperature polymeric material, part of the same Poly Aryl Ether Ketone (PAEK) family would be preferable in certain applications. This study presents the optimisation and characterisation of Poly Ether Ether Ketone (PEEK), a polymer which is part of the PAEK family with a $30^{\circ} \mathrm{C}$ lower melting temperature than PEK. The systematic characterisation of laser sintered samples of PEEK revealed a very good overall performance in comparison with the HP3 PEK material, with no change in storage modulus and only $25 \%$ drop in tensile strength. The possibility of variable building configurations available within the HT-LS system, i.e. reduced, half and full chamber building modes, is examined in relation to the mechanical performances of the components. The effect of the post sintering time, an additional heating phase supplied to the powder bed at every layer, found only in the HT-LS system EOSINT P 800, is also examined.
\end{abstract}




\section{Graphical abstract}

HT-LS of PEEK: variable size build chamber mode
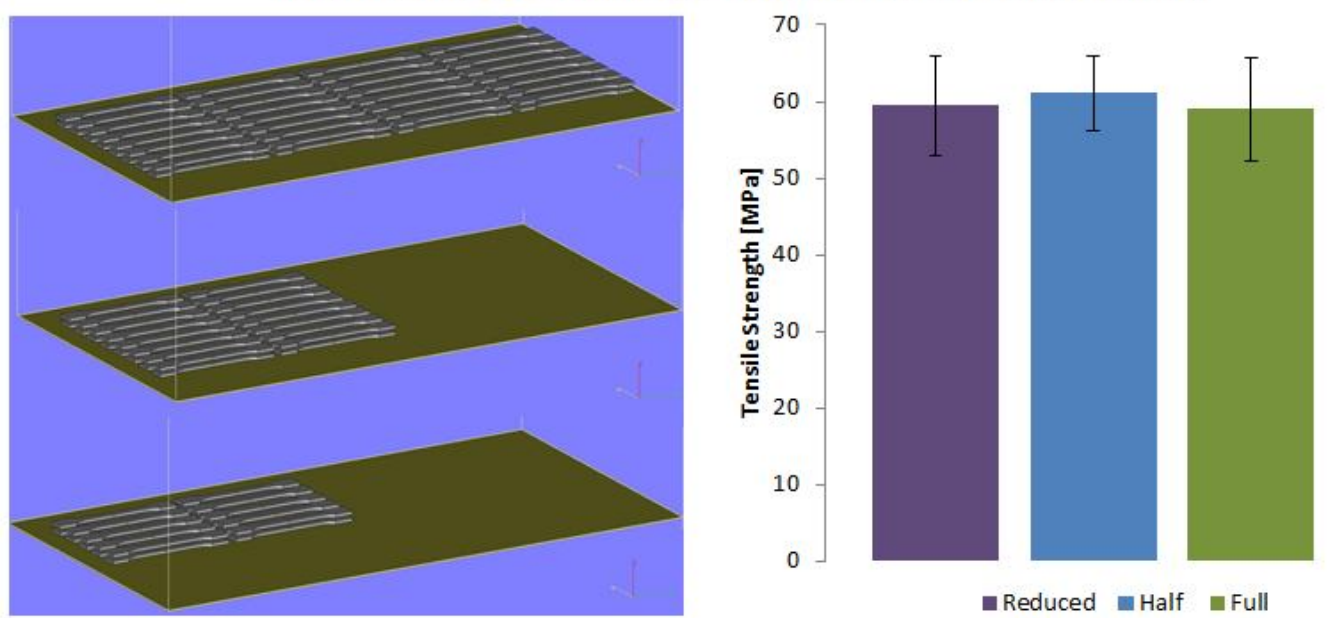

Surface morphology of HT-LS PEEK ten layer specimens
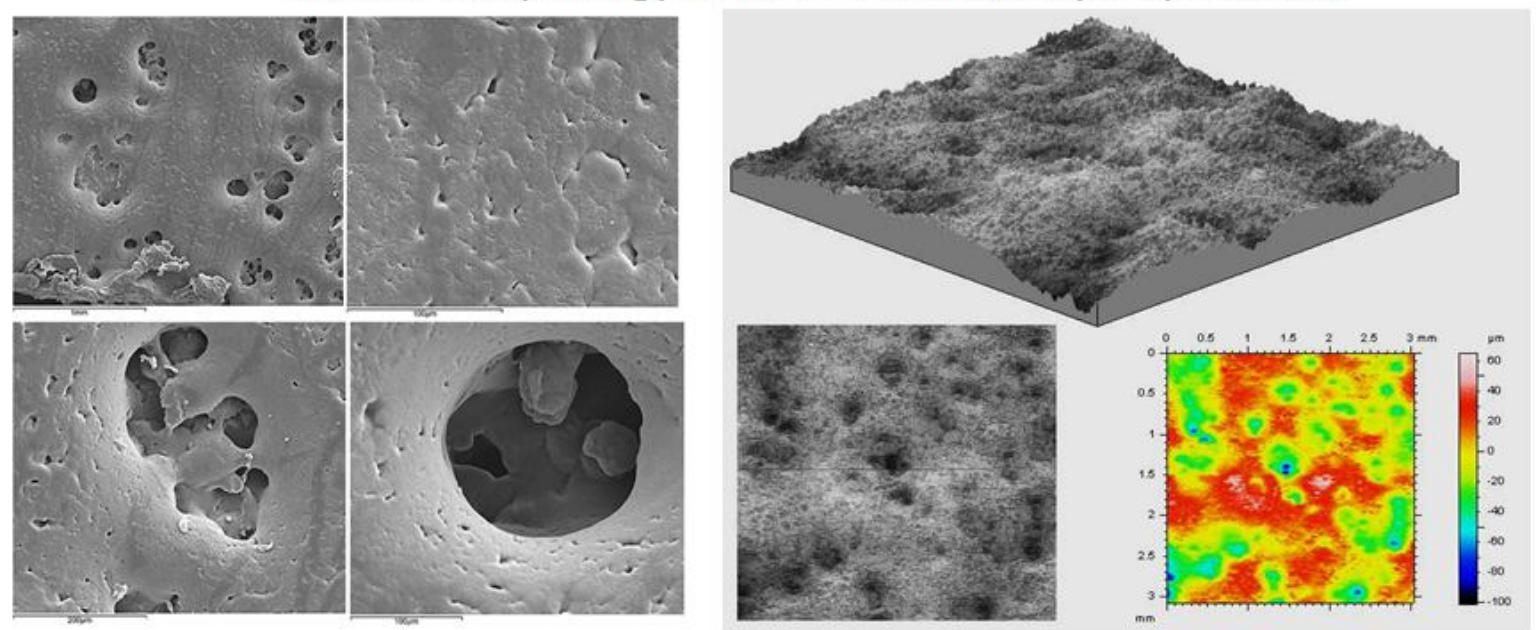

\section{Keywords}

PEEK; Laser Sintering; PEK; High Temperature; M echanical; LS

\section{Introduction}

Laser sintering (LS) or powder bed fusion is an additive manufacturing process where a laser consolidates, layer upon layer, powdered materials in order to build three dimensional objects, prototypes or fully functional parts. The high freedom of design available with LS has opened the way to a level of end-user customisation never achieved before and to the optimisation of physical components, i.e. complex light weight parts whose original functional requirements are not compromised. Because of these characteristics, industries such as aerospace, automotive and medical, have become increasingly interested in LS manufacturing [1]. However, most laser sintering systems have been developed for the processing of low temperature polymers such as nylon, which, although successful in the 
consumer goods industry, struggle to be suited for more demanding applications in the aerospace or automotive fields because of its low mechanical properties.

High Temperature Laser Sintering (HT-LS) operates at temperatures above $250^{\circ} \mathrm{C}$ and therefore can sinter high temperature thermoplastics such as Poly Aryl Ether Ketones (PAEKs). PAEKs are semicrystalline polymers with high mechanical properties, low wear factor, excellent chemical resistance, high thermal stability and also approved biocompatibility for some grades [2]. At present, the only commercial sintering system developed for high temperature polymers is the EOSINT P 800 (EOS, Germany [3]), which processes Poly Ether Ketone (PEK) powder commercially known as "EOS PEEK HP3" with melting temperature of $373^{\circ} \mathrm{C}$. Although the HP3 powder is traded under the name of "HP3 PEEK" powder according to EOS website [3] for marketing purposes, the actual material is PEK. This has been confirmed by previous publications [4] and thermal analysis data [5]. Therefore, in this study, the authors will correctly refer to HP3 as "HP3 PEK" powder.

Previous attempts of obtaining high performance parts were carried out using PEEK within in-laboratory made sintering machines, whose thermal stabilities may be questionable. Tan et al. [6-8] sintered scaffolds for tissue engineering utilising a high laser power on PEEK powder (Victrex, UK [9]) within the nylon sintering system Sinterstation 2000 (3D Systems, USA [10]). Although the lack of mechanical characterisation of the sintered specimens, the authors report on successful biocompatibility (in vitro) results in the samples containing hydroxyapatite. Rechtenwald et al. [11] modified the laser sintering nylon 12 EOSINT P 380 (EOS, Germany [3]) with an in-lab made additional structure called "heating dome", located within the building chamber. According to the authors, the structure enabled the system to reach a building temperature of $350^{\circ} \mathrm{C}$. PEEK structures were successfully sintered. However, the authors pointed out that a higher preheating temperature (temperature within the building chamber before the laser exposure) than the one achievable with the heating dome would be necessary for obtaining parts with better mechanical performances. Pohle et al. [12] provided new findings in the use of PEEK for tissue engineering applications by sintering discs made with different blends of materials. M ixtures including carbon black (Evonik, Germany [13]) to improve the flow ability and $\beta$-tricalciumphosphate ( $\beta$-TCP) to enhance the bioactivity of the sintered parts, were utilised. Von Wilmowsky et al. [14] extended the work of Pohle et al. [12] by adding bioglass in the PEEK blends. Cell morphology, cell viability and cell proliferation were examined on the sintered specimens for a longer period through in-vitro tests. All samples exhibited cell proliferation; the carbon black seemed not to affect the in vitro osteoblast behaviour; bioglass led to the highest osteoblast growth and $\beta$-TCP demonstrated a significantly lower proliferation at any time of the survey. Later on, the same group [15] sintered PEEK implants containing $\beta$-TCP and carried out in-vivo tests on animal (pigs) for the first time. The LS implant containing $\beta$-TCP exhibited the best results in terms of osseointegration, biocompatibility, bioactivity and shear polymer bone interface strength. Interestingly, the behaviour of $\beta$-TCP in vivo was much better than in the in-vitro conditions. Wegner et al. [16] and Woicke et al. [17, 18] 
successfully sintered PEK, PEEK 450PF and PEEK 150PF (Victrex, UK [9]) mixed with carbon black in different percentages, but within a very primitive equipment where layers were manually dispensed on an open air flat surface and one layer specimens were consolidated by using a Nd: YAG laser equipped with a scanner module. Kroh et al. [19] sintered samples of PEEK 150PF and 450PF (Victrex, UK [9]). The authors investigated Young's modulus and tensile strength of the samples and analysed the effect of an additional heating phase after the LS on the mechanical properties of LS PEEK 450PF samples. These latter specimens showed higher tensile strength and strain and for the additional heating time of 80 seconds the mechanical properties of the LS samples were almost comparable with those of injection moulded samples of the same material. Although the results are promising this work lacks any description of the LS system utilised. An additional heating phase of 80 seconds per layer, as proposed by Kroh et al. [19], could negatively affect the whole thermal histories of LS specimens or the geometrical accuracy of potential components. However these effects are not discussed or commented upon.

A systematic characterisation of laser sintered PEK HP3 samples manufactured in the EOSINT P 800 has been recently provided. Beard et al. [20] analysed 16 standard-shaped dog bones in terms of topology, microstructure, thermal stability and mechanical performances. The authors reported high thermal and mechanical properties (tensile strength above $90 \mathrm{M} \mathrm{Pa}$ ) and part density independent from the building position of the samples within the building chamber. Ghita et al. [21] examined the mechanical, shrinkage and degradation characteristics of PEK HP3 samples manufactured along $X, Y$ and $Z$ axis of the $P 800$ system. A higher shrinkage for samples built in the middle of the building chamber of the system and a drop of the tensile properties for samples built along the $Z$ direction were presented. In another work, Ghita et al. [4] sintered and studied for the first time HP3 PEK samples blended with used HP3 PEK powder (material that had already been through the manufacturing process). Both raw powders and sintered specimens were analysed. Although used powder showed higher melting and crystallisation temperatures, a satisfactory sintering stage between used and virgin particles was shown. Tensile strength, thermal properties, surface roughness and microstructure of the sintered components were investigated. Interestingly, the specimens incorporating used HP3 PEK powder (30\% used / $70 \%$ virgin PEK powder, weight percentage) showed a drop of the tensile strength values of only $17 \%$. However, a high level of thermo-oxidative degradation was noticed in the HP3 PEK powder due to the high processing temperature required for the PEK material and the long residence time of the powder in the LS process. Thermal-oxidative degradation is one of the major challenges for high temperature additive manufacturing and providing PEEK as a new high temperature polymer that can be processed at $20-30{ }^{\circ} \mathrm{C}$ lower than PEK HP3 can have a huge impact on the future developments in HT-LS.

This paper describes the first systematic investigation of a new member of the PAEK family for laser sintering in the only commercial HT-LS system, EOSINT P 800. The optimum processing conditions for PEEK and the unique characteristics of the $P 800$ system, such as 
variable building chamber size and post sintering time duration, are examined in order to provide a better understanding of the HT-LS process overall.

\section{Manufacturing of samples}

\subsection{Materials}

The material used for the manufacture of PEEK components is PEEK 450PF, supplied by Victrex [9] in powder form with average size of $50 \mu \mathrm{m}$. The material presents a glass transition temperature of $143^{\circ} \mathrm{C}$, a melting temperature of $343^{\circ} \mathrm{C}$ and a melt viscosity of $350 \mathrm{~Pa}$.s. The Angle of Repose (AoR), a measurement of a material flow performance, of PEEK 45OPF resulted equal to $53 \pm 1^{\circ}$ [22]. This value was higher than the AoR found for other commercial grades of LS powders like nylon and HP3 PEK (value of $38 \pm 1^{\circ}$ and $42 \pm 1^{\circ}$, respectively [22]), indicating a poorer flow. Hence, a thermal treatment was carried out on PEEK 450PF in order to improve the particle flow and shape. The powder was kept at $250^{\circ} \mathrm{C}$ for 24 hours in an air-ventilated oven, left to cool down naturally and sieved. The flow of the treated powder improved significantly with a decrease of the AoR to $46 \pm 1^{\circ}$.

\subsection{Laser Sintering process within EOSINT P 800}

The EOSINT P 800 system has a central building chamber of size $700 \times 380 \times 560 \mathrm{~mm}$, two side powder feeder bins at the top and two side overflow powder collector bins at the bottom. The machine hardware and setup is similar to standard nylon systems except the specific features of variable building chamber and post sintering phase which are unique in the HT-LS system. The P 800 is able to build components using full, half or a third of the building area (Figure 1) due to the properties of the unsintered PAEK particles that, once exposed at high temperature for a relatively long amount of time, retain a consolidated powder bed arrangement without the presence of a physical frame. 


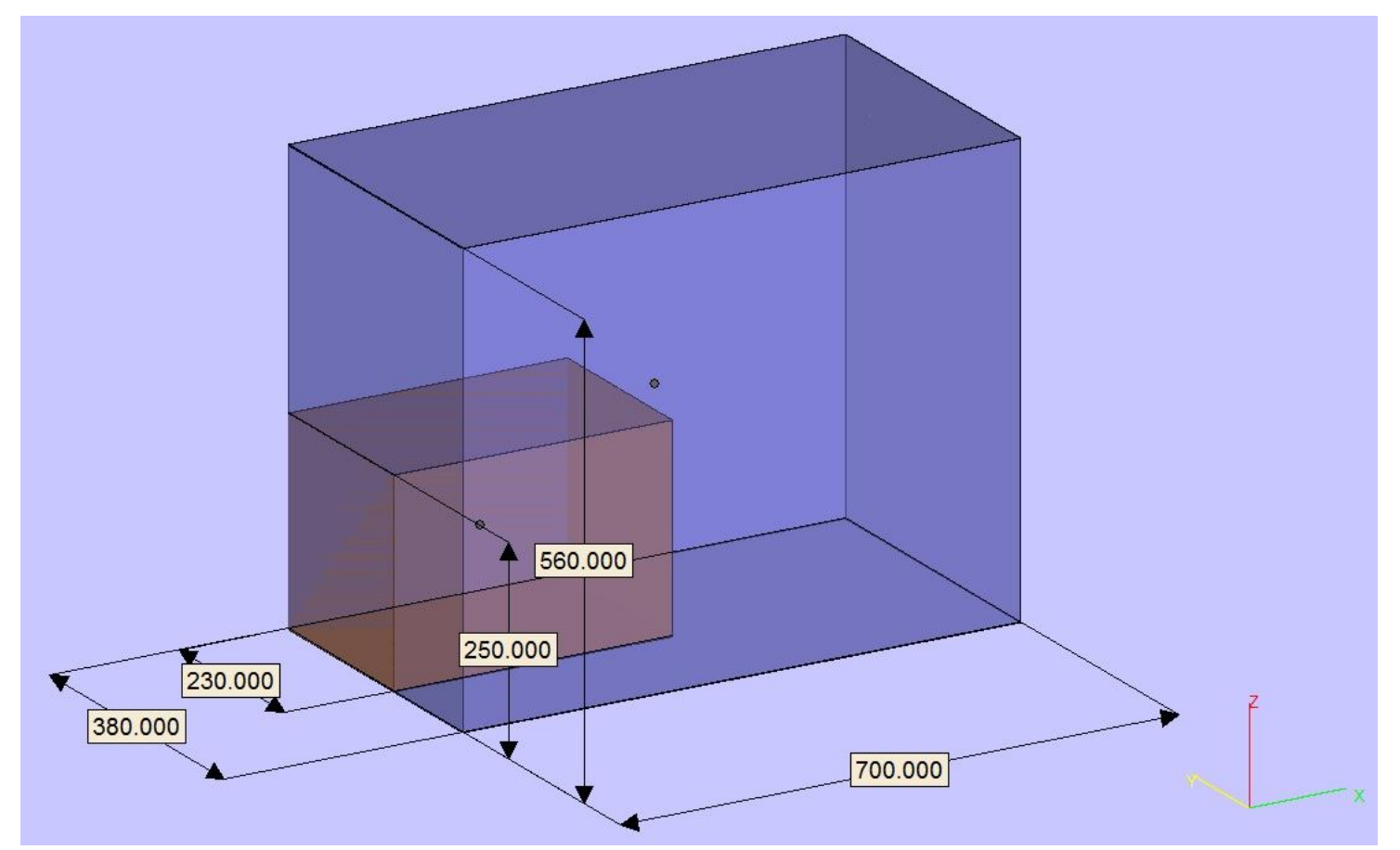

Figure 1. Building Chamber for Full and Reduced building mode in the P 800 system. Unit of measure: $\mathrm{mm}$.

A building operation comprises of three phases: warm up, actual building and cooling. During the build, as the laser exposure is completed, before the powder bed is lowered and a new layer is deposited, the heaters provide an additional heating period called "Post sintering" [23].

\subsection{Building modes}

The $P 800$ system is the only LS machine that offers variable building chamber size. The manufacturing modes called "Reduced-", "Half-" and "Full-chamber" present cross sections of $700 \times 380 \mathrm{~mm}, 350 \times 380 \mathrm{~mm}$ and $350 \times 250 \mathrm{~mm}$, respectively. Few adjustments (recoater, feeder bin, infrared heater orientation and physical switch on the system) are required in order to turn the system around the three building conditions. The effect of the building chamber size on the mechanical properties of PEEK specimens has been evaluated by manufacturing tensile testing bars in the three different modes (Figure 2). 


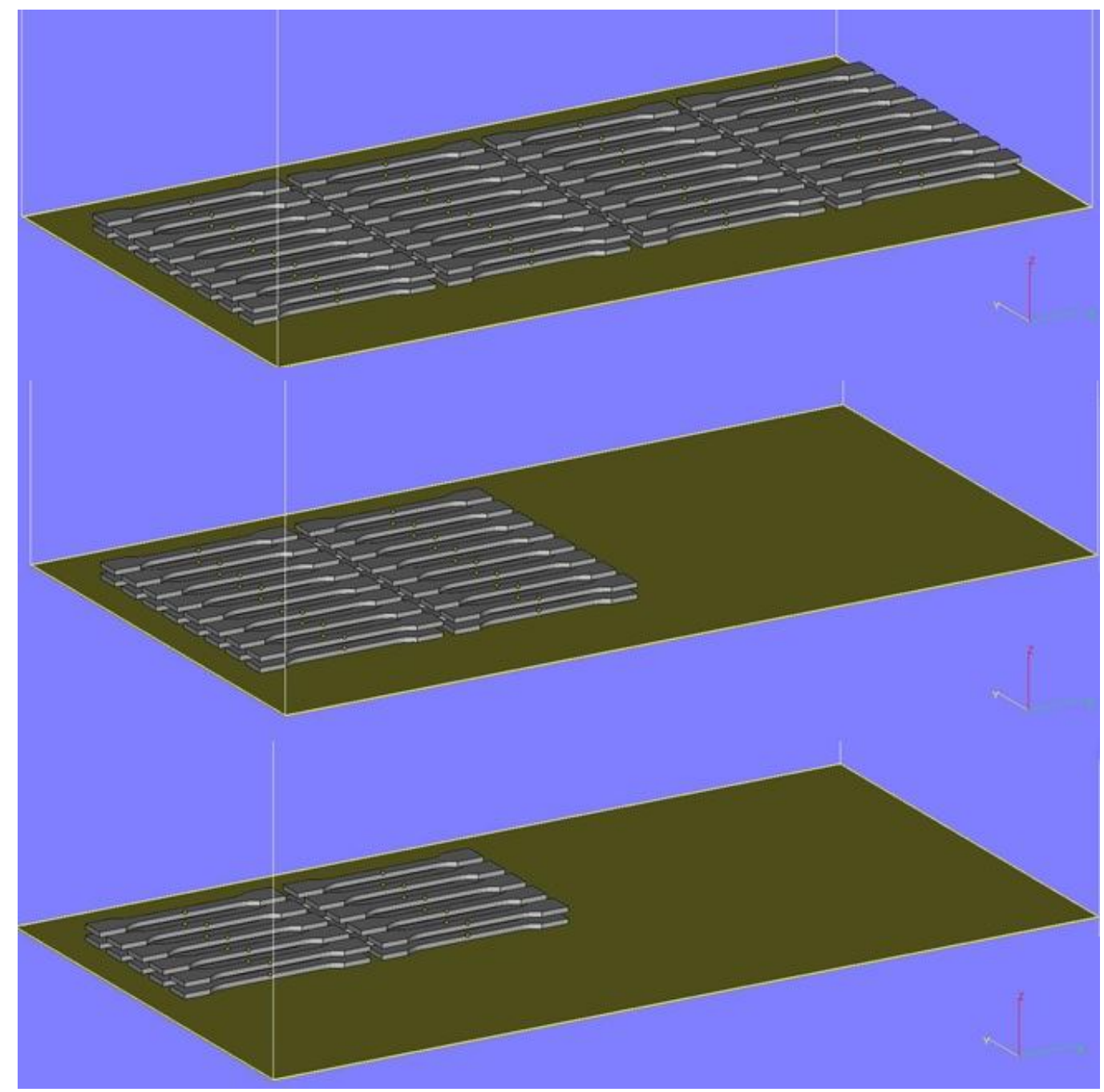

Figure 2. From the top, Full-, Half- and Reduced-chamber builds

The three builds were set with the following processing parameters: laser power of $6.8 \mathrm{~W}$ with scan speed of $1000 \mathrm{~mm} / \mathrm{s}$ for the contour exposure, laser power of $12 \mathrm{~W}$ with scan speed of $2550 \mathrm{~mm} / \mathrm{s}$ and scan spacing of $0.2 \mathrm{~mm}$ for the hatching exposure.

\subsection{Energy density}

The energy density (ED) is the amount of energy applied by the laser for sintering the material particles. It is a function of laser power (P), scan speed (v) and laser scan spacing (SS) (Eq. 1) [24].

$$
E D=\frac{P}{v \cdot S S}
$$

Equation 1. Energy density 
In order to optimise the LS process for PEEK, an identical build (the same number of parts and the same configuration) has been performed at different values of ED. The manufacturing parameters and the energy density values calculated according Eq. 1 are listed in Table 1. Laser speed and scan distance were not varied and kept constant at 2550 $\mathrm{mm} / \mathrm{s}$ and $0.2 \mathrm{~mm}$, respectively.

\begin{tabular}{|c|c|c|}
\hline $\begin{array}{c}\text { Processing } \\
\text { parameters profile }\end{array}$ & $\begin{array}{c}\mathrm{V}=2550 \mathrm{~mm} / \mathrm{s} \\
\mathrm{SS}=0.2 \mathrm{~mm} \\
\text { Laser Power [W] }\end{array}$ & ED $\left[\frac{\mathbf{J}}{\mathbf{m m}^{\mathbf{2}}}\right]$ \\
\hline Build 1 & 7.5 & 0.015 \\
\hline Build 2 & 9.0 & 0.018 \\
\hline Build 3 & 12.0 & 0.024 \\
\hline Build 4 & 13.5 & 0.026 \\
\hline Build 5 & 15.0 & 0.029 \\
\hline Build 6 & 16.5 & 0.032 \\
\hline
\end{tabular}

Table 1. Processing parameters and corresponding ED

The upper and lower limit of energy density (builds 1 and 6) leading to specimens able to be tested, i.e. solid enough to be tested and not degraded during the laser scanning, were previously identified by running a qualitative build of specimens with individual settings of energy density values. Such settings covered the whole spectrum of energy densities safely applicable to the system.

\subsection{Post sintering}

In order to obtain a homogenous molten film for parts exposed in the same layer, the EOSINT P 800 is configured with an additional heating period before the spreading of a new fresh layer of powder [23]. In this phase, called "Post Sintering (PS)", the infrared heaters above the building chamber are all turned on for 12 seconds to guarantee uniform melting between the regions exposed first and last in the exposure sequence. Interestingly, this phase is not present in $\mathrm{LS}$ standard machines treating other materials like nylon. Although set at $12 \mathrm{~s}$, this parameter can be user defined. 


\section{Experimental methods}

\subsection{Tensile testing}

Tensile samples were manufactured with ISO 527-2-1A geometries along the $x$ direction of the referencing system within the building chamber. Tensile testing experiments were performed by using a LLOYD instruments EZ 20 mechanical testing machine at room temperature $\left(20^{\circ} \mathrm{C}\right)$. The testing speed was equal to $2 \mathrm{~mm} / \mathrm{min}$ and the gauge length was 85 $\mathrm{mm}$. A minimum of 20 samples were manufactured and tested for every build. The average ultimate tensile strength and standard deviation were calculated.

\subsection{Post sintering samples: one and ten layer specimens}

PS samples were $X Y$ cross sections (Figure 3 ) of the tensile samples manufactured with ISO 527-2-1A geometries along the $x$ direction of the referencing system within the building chamber produced with the processing parameters corresponding to build 5 and different PS times.

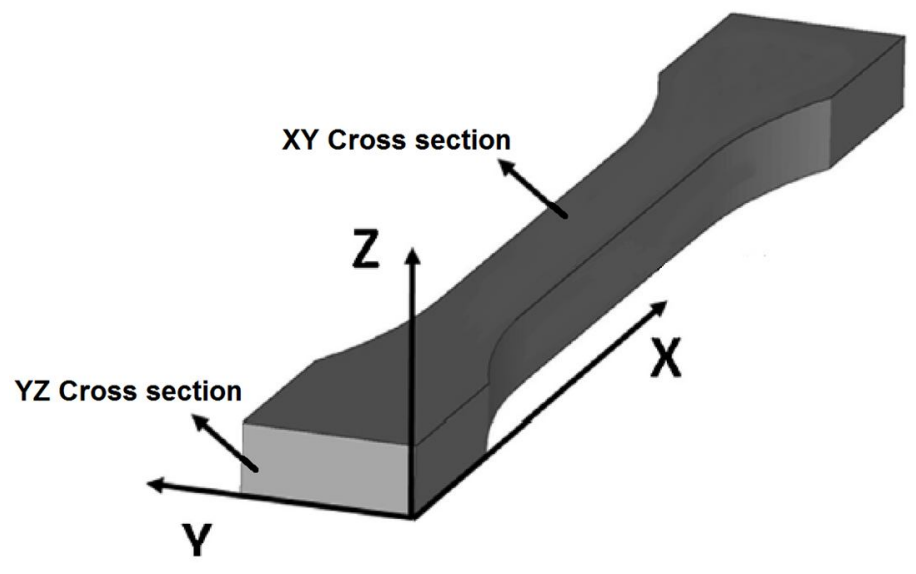

Figure 3. $X Y$ cross section of one and ten layer samples. $Y Z$ cross section used for imaging layer bonding properties

One layer specimens were tensile samples exposed only once to the laser followed by the post sintering phase. Once one exposure was completed, the process was manually interrupted and the samples left to cool down naturally. Ten layer specimens were tensile bars exposed to the laser, followed by the post sintering and the recoating phases, ten times. The purpose of the production of these specimens was to understand the effect of the post sintering time: it was expected that looking at the molten and solidified surface of one layer and ten layer would give an indication of the importance of this heating phase.

One and ten layer samples in PEEK 450PF were manufactured with PS times of $6 \mathrm{~s}, 9 \mathrm{~s}, 12 \mathrm{~s}$ and $15 \mathrm{~s}$. Similarly one and ten layer samples in PEK HP3 produced according to 
manufacturing parameters of build 5 and with standard recommended PS of $12 \mathrm{~s}$ were built and used as benchmark for the PEEK samples.

\subsection{Scanning Electron M icroscopy (SEM)}

SEM examination was carried out by using a Hitachi S - 3200N scanning electron microscope. The samples were coated with $10 \mathrm{~nm}$ of gold/palladium in order to reduce the surface charging. The electron secondary imaging was set with an accelerating voltage of 25 $\mathrm{kV}$. Both one and ten layer specimens of PEEK 450PF and PEK HP3 were analysed using SEM . In order to investigate the layer bonding properties as function of the post sintering time the ten layer samples were cooled down with liquid nitrogen and manually snapped. The fracture surface (YZ cross sections in Figure 3) was then analysed with the SEM according the setting described above.

\subsection{Surface morphology measurements}

The surface morphology of laser sintered one and ten layer specimens was evaluated using a Taylor-Hobson Talyscan 150 surface profiler. An area of $3 \mathrm{~mm} \times 3 \mathrm{~mm}$ was scanned on the $X Y$ surface of each sample at $1000 \mu \mathrm{m} / \mathrm{s}$ with spacing along the $X$ axis of $3 \mu \mathrm{m}$ and of $5 \mu \mathrm{m}$ along the $Y$ axis. The evaluation of the descriptor surface roughness $\left(S_{a}\right)$ and the imaging of surface profiles were performed using the software Talymap.

\subsection{Dynamic Mechanical Analysis (DMA)}

DM A allows the study of the viscoelastic behaviour of a given material by the measurement of the complex modulus. While the real part of the complex modulus represents the elastic behaviour (Storage modulus E') of a material, the imaginary part (Loss modulus, E') represents its viscous performance. The tests were carried out using the M ETTLER TOLEDO DM A - 1. All the specimens were built with nominal dimension of $30 \mathrm{~mm} \times 7 \mathrm{~mm} \times 2 \mathrm{~mm}$ and tested in three-points-bending mode (Frequency $1 \mathrm{~Hz}$ and strain displacement $10 \mu \mathrm{m}$ ). During the test, each sample was heated from $30^{\circ} \mathrm{C}$ to $300{ }^{\circ} \mathrm{C}$ at heating rate of $3{ }^{\circ} \mathrm{C} / \mathrm{min}$. A minimum of four repeats were carried out for each type of processing conditions.

\subsection{Micro-Computer Tomography (M icro-CT)}

In order to investigate the internal structure, samples built at different manufacturing settings (cubic samples of approximately $5 \mathrm{~mm} \times 5 \mathrm{~mm} \times 5 \mathrm{~mm}$ ) were analysed by micro-CT. The tests were carried out using an X-Tek Bench top CT 160 Xi System with resolution of 3 $\mu \mathrm{m}$. Samples were examined at $65 \mathrm{kV}$ and 82-90 $\mu \mathrm{A}$. The data were then reconstructed using the software CT Pro 3D [25] and imaged with the software VG Studio Max 2.1 [26]. 
The samples were investigated qualitatively and quantitatively by estimating the pore size and the material density.

\subsection{Statistical analysis}

The software package IBM SPSS Statistics version 21 [27] has been used for evaluating the statistical differences between data groups of samples built with different manufacturing parameters. Experimental results have been analysed using the post-hoc analysis of the ANOVA with Tamhane's T2 at a confidence level of $95 \%$. The statistical results are presented in terms of ' $P$-Values'. Any P -value found smaller than 0.05 means that there is a significant difference between the compared groups of data. Significant difference indicates that the variation of the means values between two or more datasets is not due to the natural variation of the results, but to the conditions with which the data was generated.

\section{Results}

\subsection{Full-, Half- and Reduced-Chamber building modes}

A standard powder bed LS system operates by spreading layers of powder across the entire build chamber. The top of the powder bed is thermally controlled by infrared heaters located above the building chamber, while the rest of the powder bed is uniformly regulated by heaters incorporated in the frame of the building chamber. This setup should guarantee a uniform temperature distribution. However, previous research showed the presence of small thermal gradients in the building area [21,28]. Temperature discrepancies lead to part shrinkage, which, in turn, alters density and ultimately the mechanical behaviour of the components. Varying the building mode in the P 800 system means varying this condition of direct contact between the frame heating elements and powder bed. While in full-chamber mode all sides of the powder bed are in contact with heaters, half chamber mode presents only three sides directly heated and one fully exposed to the building environment atmosphere. Reduced chamber mode presents even less direct heating control as only two sides are directly heated and two sides are left exposed. It appeared therefore essential to investigate the effect of the building mode on the part properties. The tensile strength values (average value and standard deviation) of the tensile bars (Figure 2) fabricated in Reduced- , Half- and Full- Building Chamber are reported in Figure 4. 


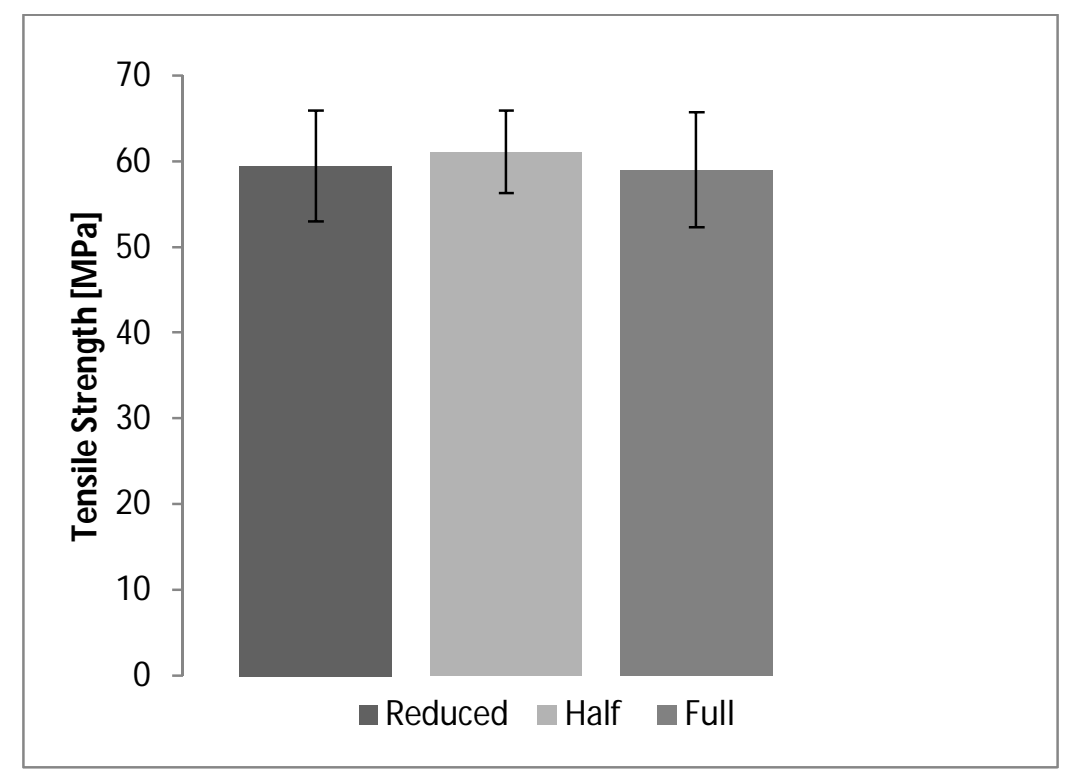

Figure 4. Tensile strength of the samples built in reduced-, half- and full-building chamber and standard deviation bars

The results displayed in Figure 4 clearly present tensile strength values independent from the building chamber mode. This shows that the energy supplied by the top infrared heaters is significantly higher and more prominent in the process than the energy provided by the frame heaters and the powder bed. This is an important finding for real manufacturing processes and applications where repeatability and accuracy are key parameters in the future uptake of a technology. These findings also allowed the authors to carry out the optimisation of the processing parameters in terms of energy density and post sintering time by using the reduced-chamber building mode.

\section{2 Optimisation of the energy density}

\subsubsection{Tensile testing results}

The tensile strength values of the same build manufactured at different energy densities are reported in Figure 5. 


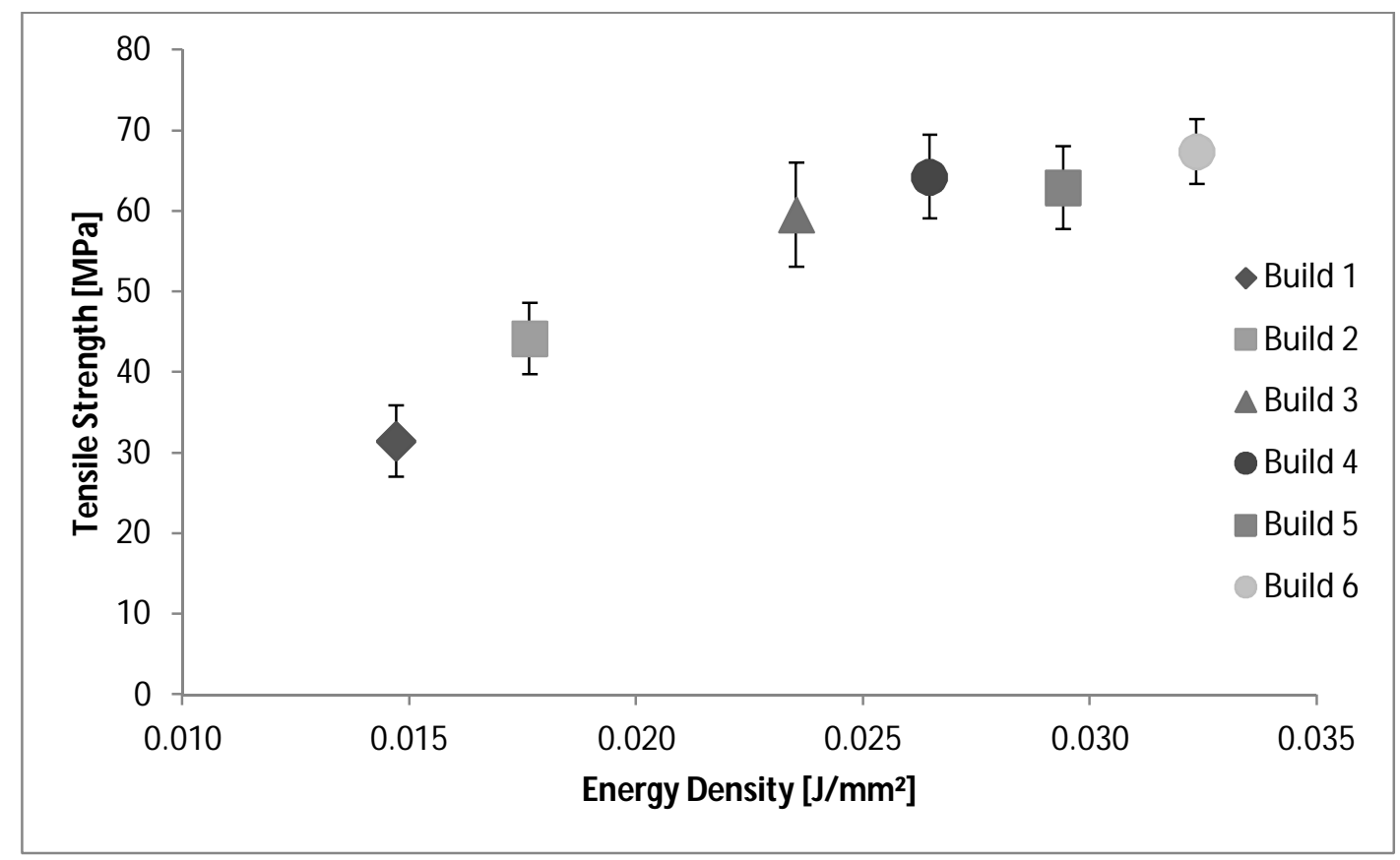

Figure 5. Tensile strength and standard deviations versus energy density

As expected, the average tensile strength values increased with higher energy densities. This was not unforeseen as similar results were exhaustively investigated for nylon-based components $[24,29,30]$. The results of the ANOVA analysis are shown in Table 2 . It is interesting to note that the tensile strength values corresponding to the energy densities $0.026,0.029$ and $0.032 \mathrm{~J} / \mathrm{mm}^{2}$ are not significantly (statistically) different which means that these three energy densities led to the similar mechanical performances in the samples. This is not surprising as similar results were found also in laser sintering of Nylon 12 [29]. Higher laser energy inputs lead to higher mechanical performances until a plateau in the mechanical properties is reached and additional laser energies no longer affect the mechanical testing results. The parameters of build 5 corresponding to the energy density of $0.029 \mathrm{~J} / \mathrm{mm}^{2}$ were then used as an optimal set for the next builds of this work.

\begin{tabular}{|l|l|l|l|l|l|}
\hline Groups & $\mathrm{P}$ & Conclusion & Groups & $\mathrm{P}$ & Conclusion \\
\hline $1-2$ & 0 & Different & $2-6$ & 0 & Different \\
\hline $1-3$ & 0 & Different & $3-4$ & 0.152 & Not different \\
\hline $1-4$ & 0 & Different & $3-5$ & 0.614 & Not different \\
\hline $1-5$ & 0 & Different & $3-6$ & 0.001 & Different \\
\hline $1-6$ & 0 & Different & $4-5$ & 0.997 & Not different \\
\hline $2-3$ & 0 & Different & $4-6$ & 0.185 & Not different \\
\hline $2-4$ & 0 & Different & $5-6$ & 0.008 & Not different \\
\hline $2-5$ & 0 & Different & & & \\
\hline
\end{tabular}

Table 2. ANOVA analysis results for tensile strength values of samples built at different manufacturing settings 
In Figure 6 , the tensile strength values found for PEEK 450PF-Build 5 has been plotted in comparison to the tensile strength value of HP3 PEK [4]. The tensile strength of PEEK 450PF is approximately $20 \mathrm{MPa}$ lower than the value found for PEK HP3, but still acceptable for a wide range of engineering applications [31]. The key is that the laser sintered PEEK parts still retain the same high operating temperature of PEK components $\left(250-260^{\circ} \mathrm{C}\right)$ [32], but provide a better processing window because of the lower melting temperature of the PEEK material.

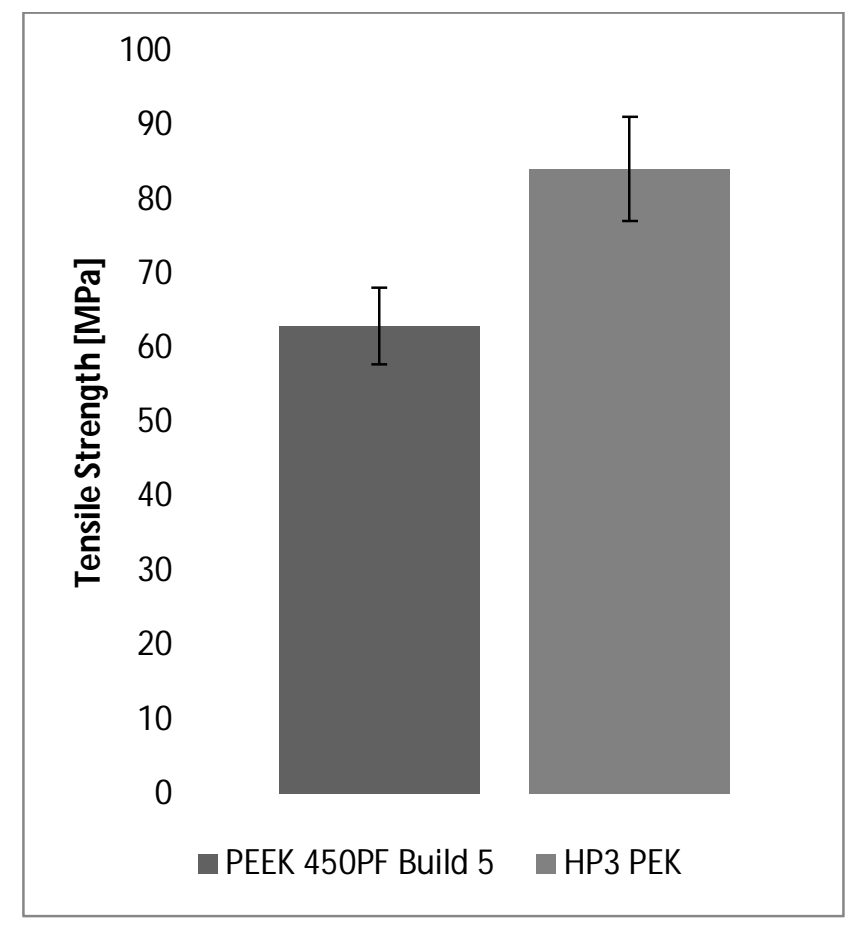

Figure 6. Tensile strength values of PEEK 450PF and HP3 PEK [21] and their standard deviations 


\subsubsection{DMA results}

Storage and Loss moduli of PEEK samples built at different energy densities and laser sintered HP3 PEK are shown in Figure 7 and Figure 8.

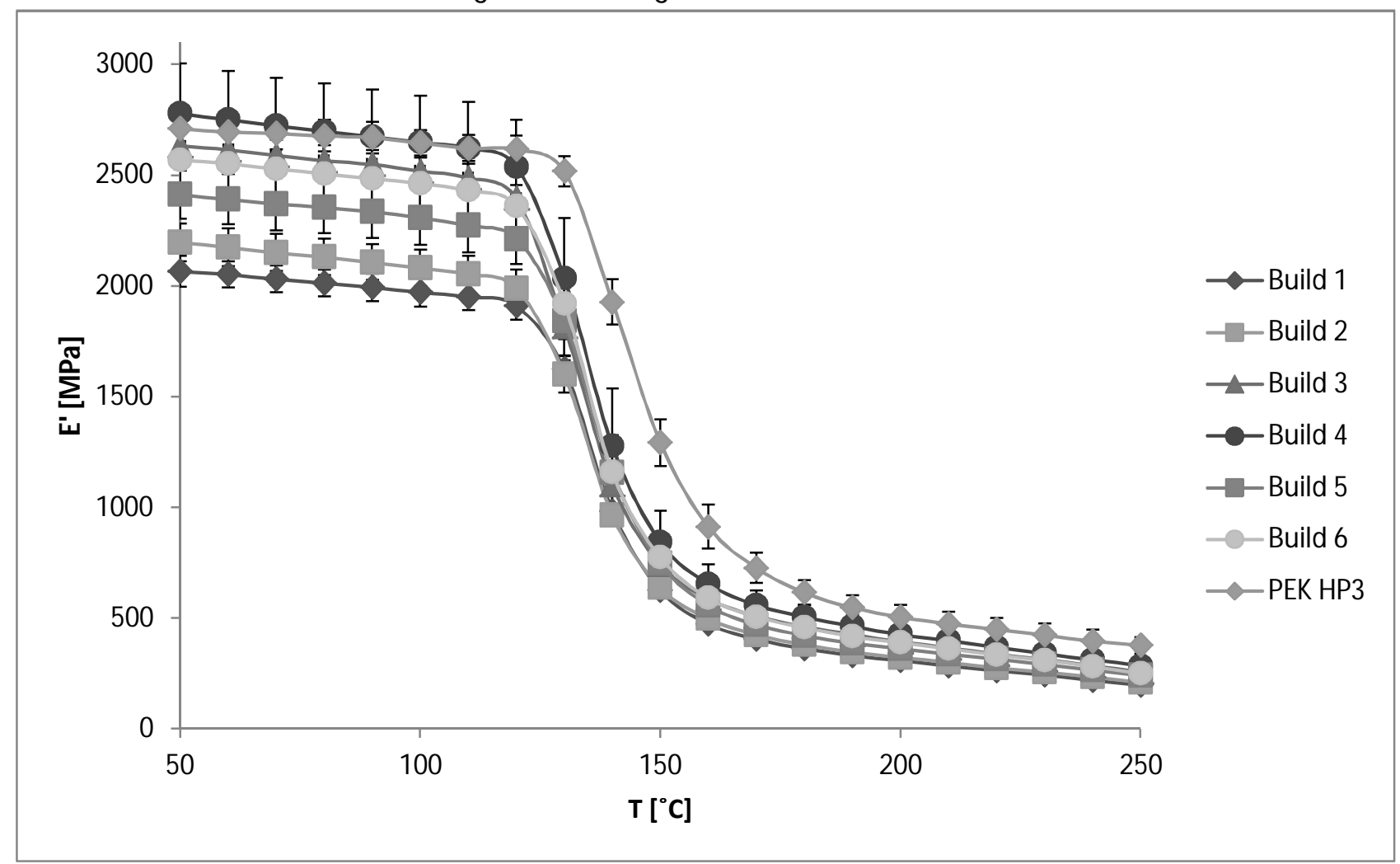

Figure 7. Storage M odulus E' for varied energy densities 


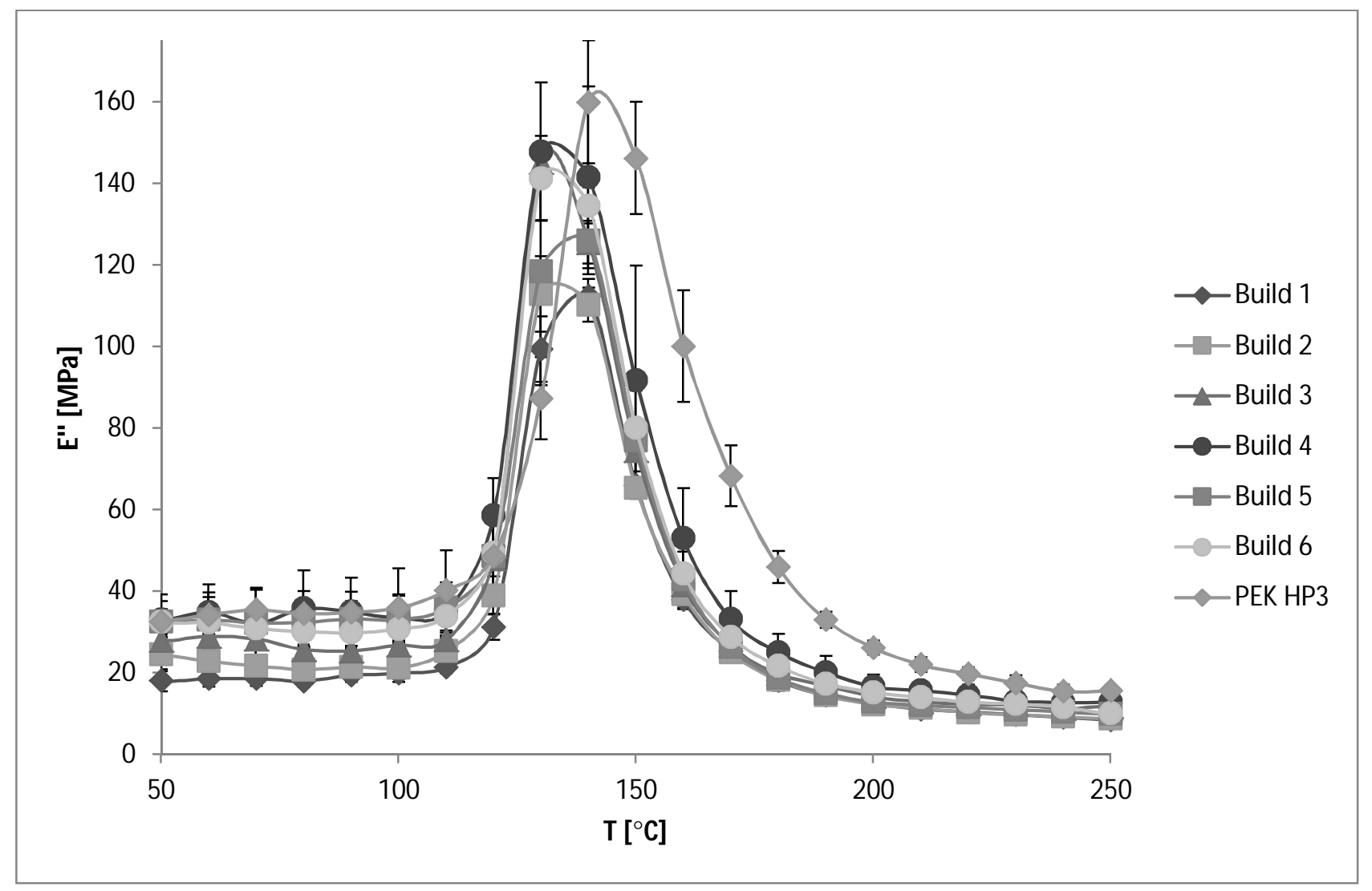

Figure 8. Loss M odulus E" for varied energy densities

The storage modulus of PEEK samples, equal to 2000-2800 M Pa in the temperature range $30-110^{\circ} \mathrm{C}$, falls drastically in proximity of the glass transition temperature $\left(140{ }^{\circ} \mathrm{C}\right)$ and stabilizes above $100 \mathrm{M} \mathrm{Pa}$ for temperatures higher than $200^{\circ} \mathrm{C}$. As seen in the tensile testing results, this mechanical modulus seems to improve with increasing energy densities, especially for the builds 1, 2 and 3. It is possible to notice that PEEK and PEK samples present the same storage profile and values, with the only difference that PEK units exhibit higher glass transition temperature.

The loss modulus of PEEK specimens results equal to 20-40 $\mathrm{MPa}$ in the temperature range $30-100{ }^{\circ} \mathrm{C}$, increases above $150 \mathrm{MPa}$ in the glass transition region $\left(110-170{ }^{\circ} \mathrm{C}\right)$ and levels off at 10-20 M Pa for higher temperatures. Also in this case, samples manufactured at higher energy densities appear to have higher peak heights. The HP3 PEK samples exhibit similar loss modulus profile, only slightly shifted at higher temperatures and higher peak height.

Both PEEK and PEK exhibit fairly high standard deviations for storage and loss moduli. This effect could be inherent to the layering structure of the samples; weaker bonds along the $Z$ axis might cause these variations. 


\subsubsection{Micro-CT results}

An example of the micro-CT imaging analysis carried out for all the PEEK samples manufactured at different energies density is reported in Figure 9. The sample was built with the processing parameters corresponding to build 5 .

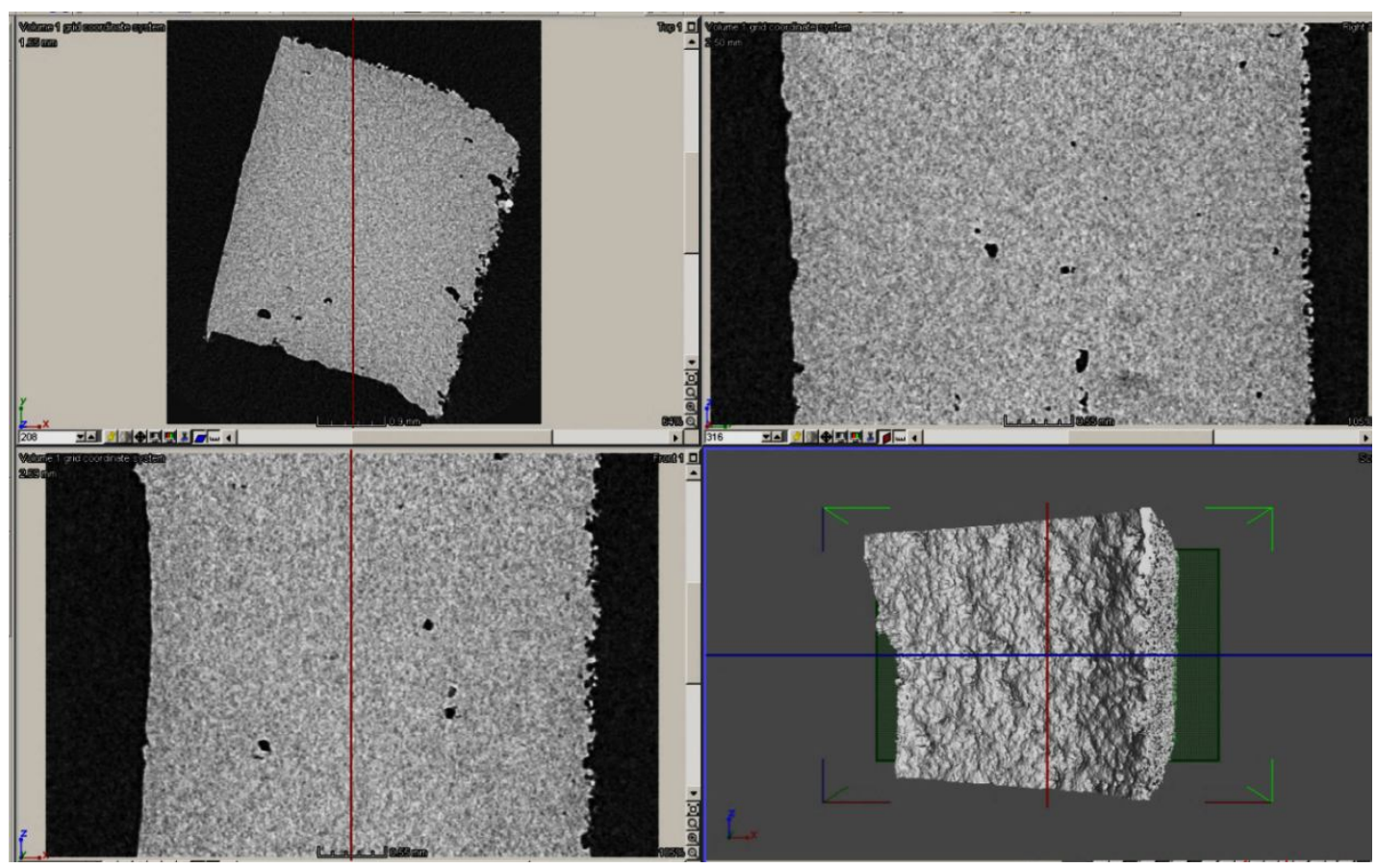

Figure 9. Build 5 sample (PS = 12 s)

Although the structures appear fairly dense, the presence of few pores with size up to $1 \mathrm{~mm}$ can be still seen. The estimates of material densities of all the samples built at varied energy densities are listed in Table 3. As seen in the tensile testing, the increase of the energy density induced a denser microstructure. The material density increased with the energy density up to the value $0.029 \mathrm{~J} / \mathrm{mm}^{2}$, where it started to decrease.

\begin{tabular}{|c|c|c|}
\hline $\begin{array}{c}\text { Processing parameters } \\
\text { profile }\end{array}$ & ED $\left[\frac{\boldsymbol{J}}{\mathbf{m}^{\mathbf{2}}}\right]$ & $\begin{array}{c}\text { Material density } \\
{[\%]}\end{array}$ \\
\hline Build 1 & 0.015 & 82.63 \\
\hline Build 2 & 0.018 & 96.88 \\
\hline Build 3 & 0.024 & 97.96 \\
\hline Build 4 & 0.026 & 95.37 \\
\hline Build 5 & 0.029 & 99.65 \\
\hline Build 6 & 0.032 & 97.91 \\
\hline
\end{tabular}

Table 3. M aterial density estimates 


\subsection{Post sintering}

\subsubsection{SEM features}

The top surface (XY cross section Figure 3) of one layer and ten layer samples was imaged with SEM in different areas for all samples. The results of the specimens manufactured with post sintering time of $6 \mathrm{~s}$ (Figure 10) and $12 \mathrm{~s}$ (Figure 11) are shown only. The standard post sintering time is $12 \mathrm{~s}$. The samples with this post sintering were compared with the samples with post sintering time of 6 seconds (half standard post sintering time) to best highlight the effects due of the post sintering phase and possibly the importance of this step within the LS process.
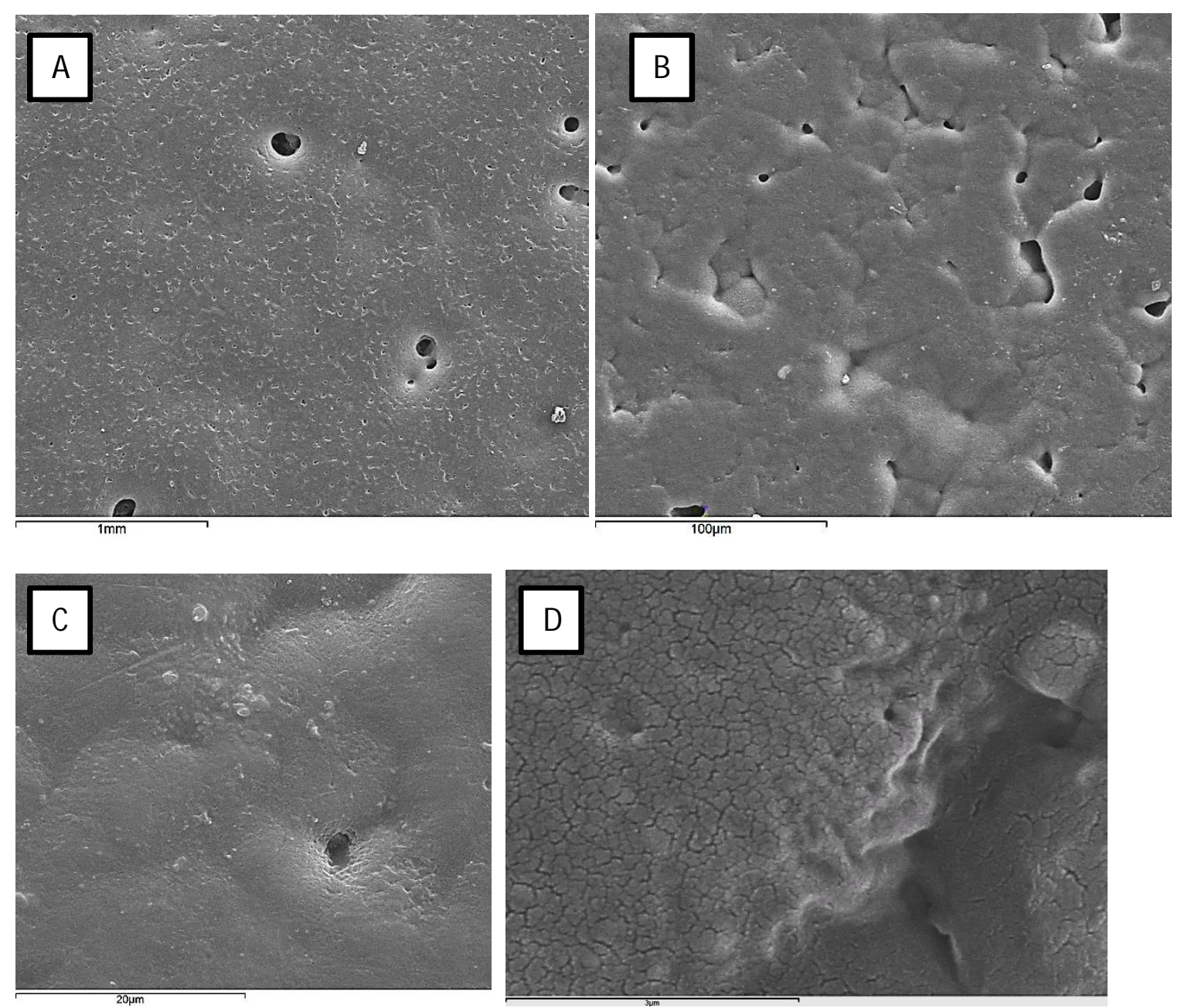

Figure 10. PEEK one layer specimen built along $x$ direction, $P S=6 s$ at varied orders of magnification 

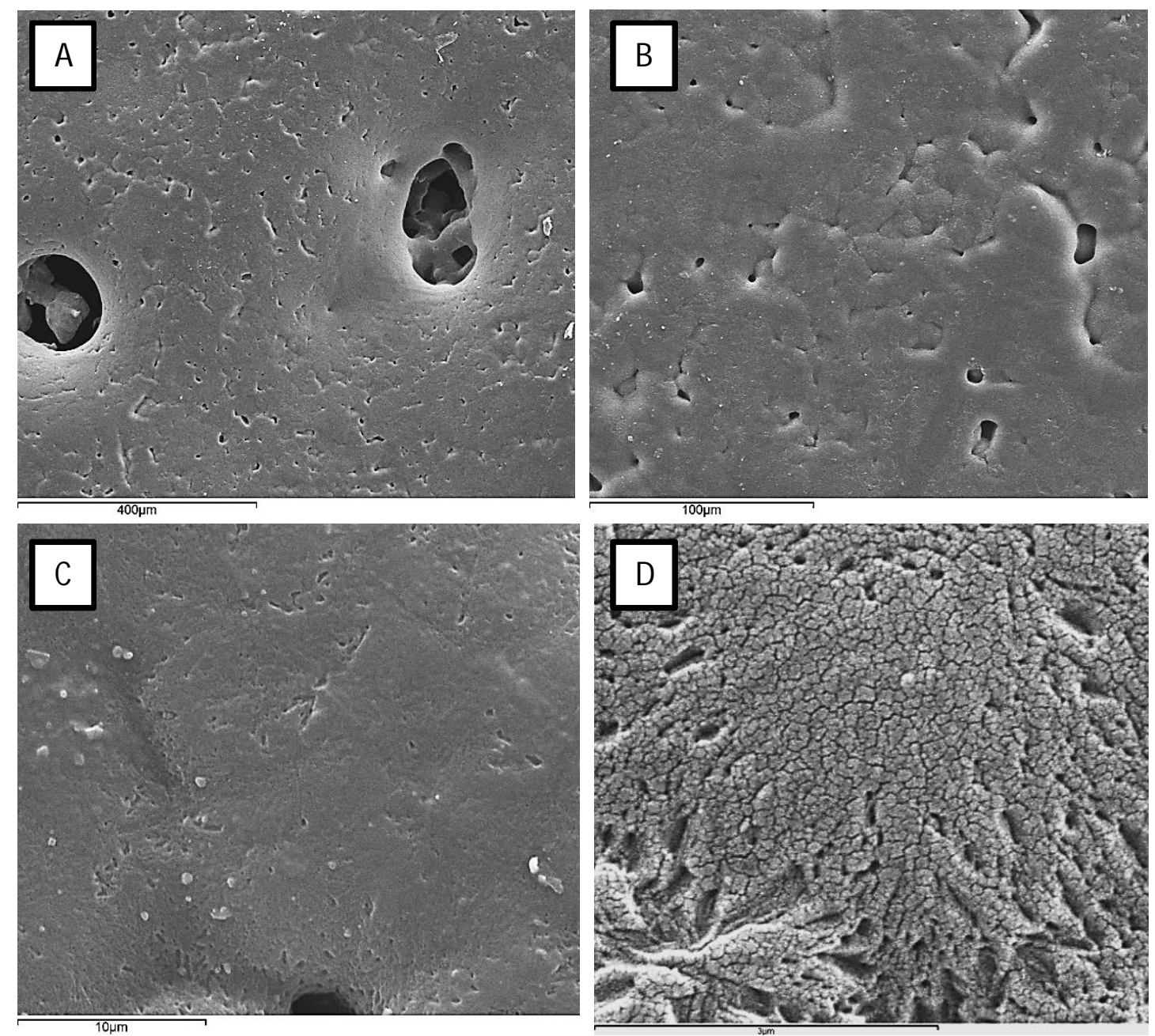

Figure 11. PEEK one layer specimens built along $\mathrm{x}$ direction, $\mathrm{PS}=12 \mathrm{~s}$ at varied orders of magnification 


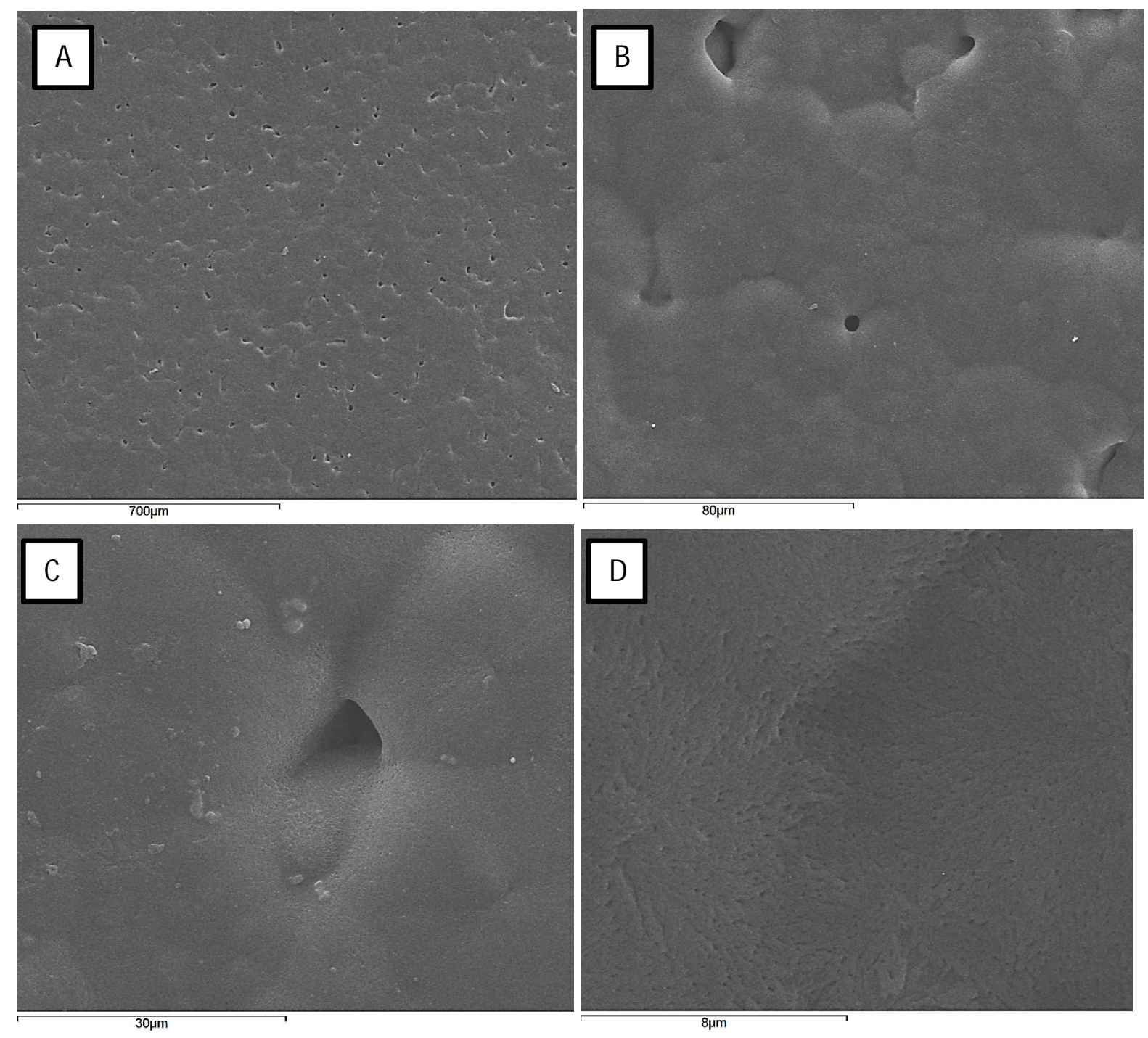

Figure 12. PEK HP3 one layer specimen, $\mathrm{PS}=12 \mathrm{~s}$, at varied orders of magnification 
The one layer PEEK specimens (Figure 10 and Figure 11) with post sintering of $6 \mathrm{~s}$ and $12 \mathrm{~s}$ do not seem to show significant distinctions. Both samples exhibit the presence of cavities of similar size. Similar surface irregularities were also found by Ho et al. [33] when optimising the $L S$ process for polycarbonate use. The authors utilised the sintering of one layer specimens as one of the methods to define the optimum energy density, expecting that a uniform layer is related to a good degree of sintering. However, the sintered layers were still presenting the same type of surface blemishes even when the laser power was set at the value leading to the highest mechanical properties in the corresponding tensile samples. It seems therefore that the presence of these irregularities will appear even at optimal processing conditions. The examination at higher orders of magnification (Figure 10 $D$, Figure $11 \mathrm{D}$ ) outlines in both cases the presence of micro cracks. This is likely to be due to the rapid cooling as the process was disrupted and forced to cool.

One layer specimens of PEK HP3 (Figure 12) did not show a homogenous molten film although the cavities were smaller than those noticed for one layer PEEK samples. It is possible that the sintering of just one layer is not enough to remove the irregularities related with the powder bed underneath.

For these reasons, ten layer samples were created and analysed. SEM captures of the ten layer specimens with post sintering time equal to 6 and $12 \mathrm{~s}$ are shown in Figure 13 and Figure 14, respectively. The PEK HP3 sample used as benchmark is shown in Figure 15. 

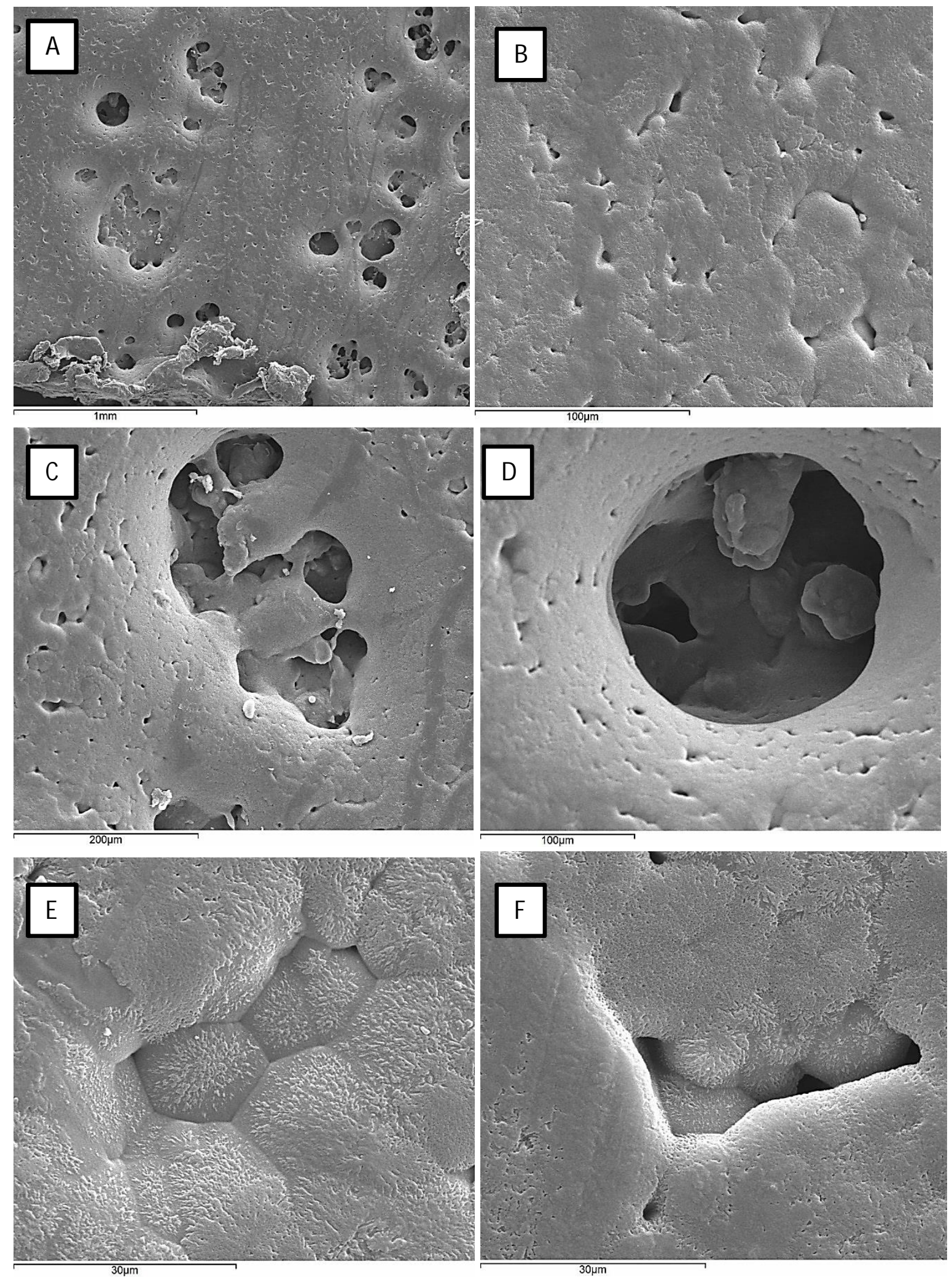

Figure 13. PEEK ten layer specimen built along $x$ direction, $P S=6 s$, varied orders of magnification 


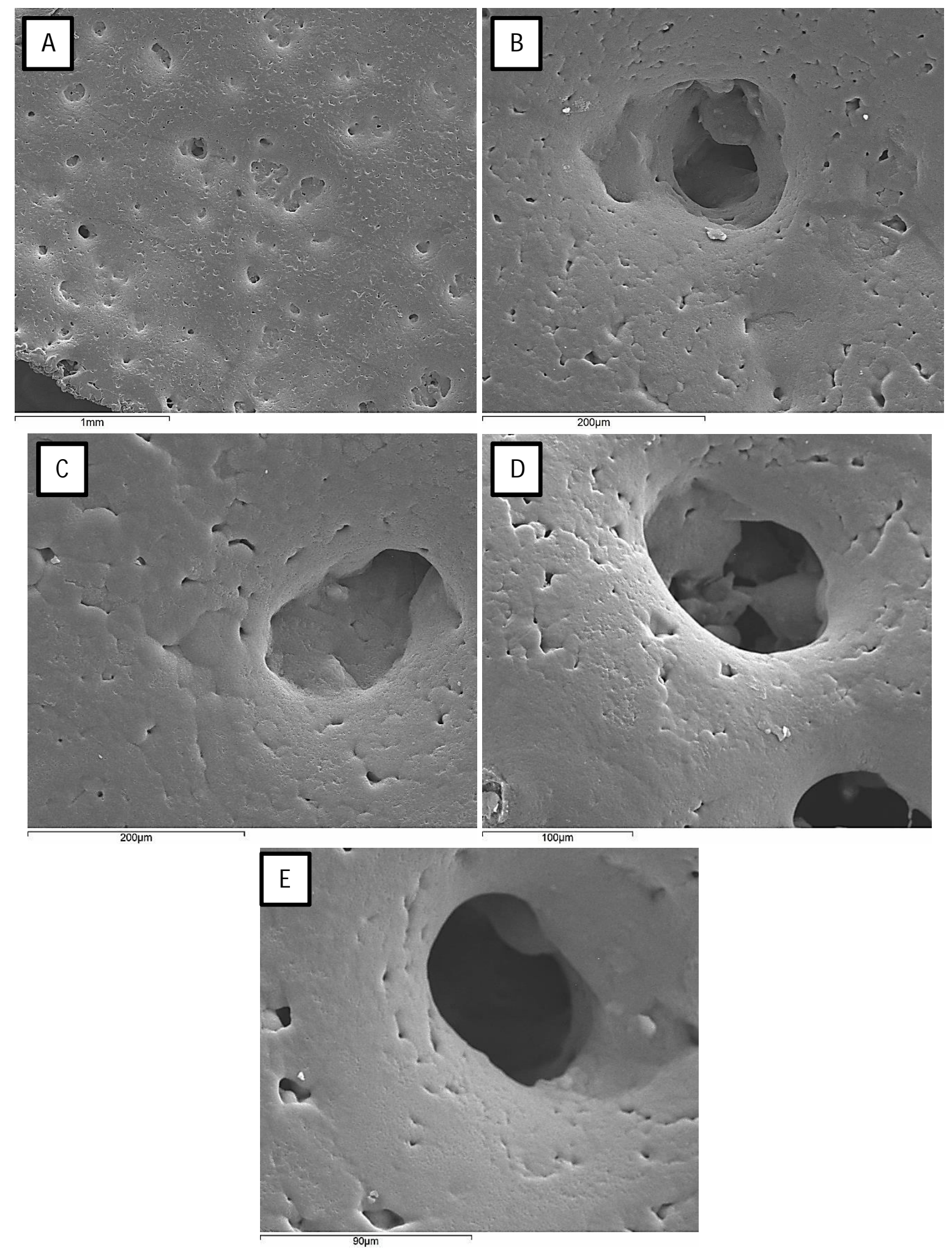

Figure 14. PEEK ten layer specimens built along $x$ direction, $P S=12 \mathrm{~s}$, varied orders of magnification 

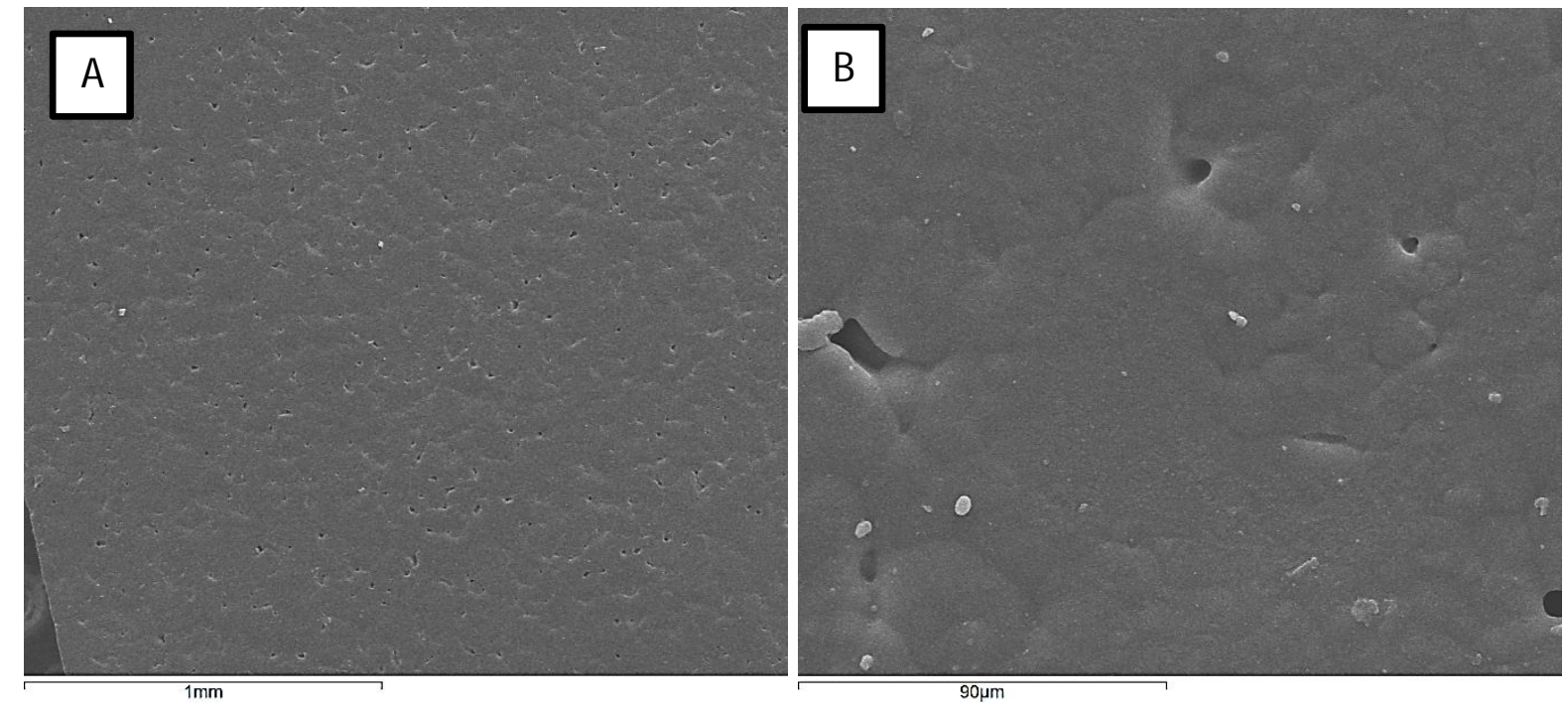

Figure 15. PEK HP3 ten layer specimen built along $x$ direction, $P S=12 \mathrm{~s}$, varied orders of magnification

Irregularities and cavities can be clearly identified for both one and ten layer PEEK specimens manufactured with post sintering times of both $6 \mathrm{~s}$ and $12 \mathrm{~s}$. Hence, 6 and $12 \mathrm{~s}$ post sintering times do not lead to the formation of uniform molten layer and consequentially fully dense parts. PEK HP3 samples (Figure 28) showed more uniform layers than PEEK, with only a few very small cavities. The cavities in the PEEK samples are fairly deep and seem to transfer from one layer to the other, although it is expected they will fill up with fresh powder. This is an interesting effect that can explain some of the porosity noticed in the parts. However, it is not clear at this stage whether the presence of the post sintering phase helps or actually creates larger and deeper cavities in PEEK samples. The presence of these cavities could also be related to the particle morphology and particle arrangement in the powder bed. Previous research has proved that the particle morphology strongly affects the flow behaviour of LS materials [22], implying that flow properties have a key role in initial stages of the LS process such as the spreading of layers and the achievement of a dense powder bed. Figure 16 shows the experimental evaluation of the particle shape descriptors roundness [34] for PEK HP3, PEEK 450PF powder before and after tempering. The method used for analysis was described elsewhere [22]. 


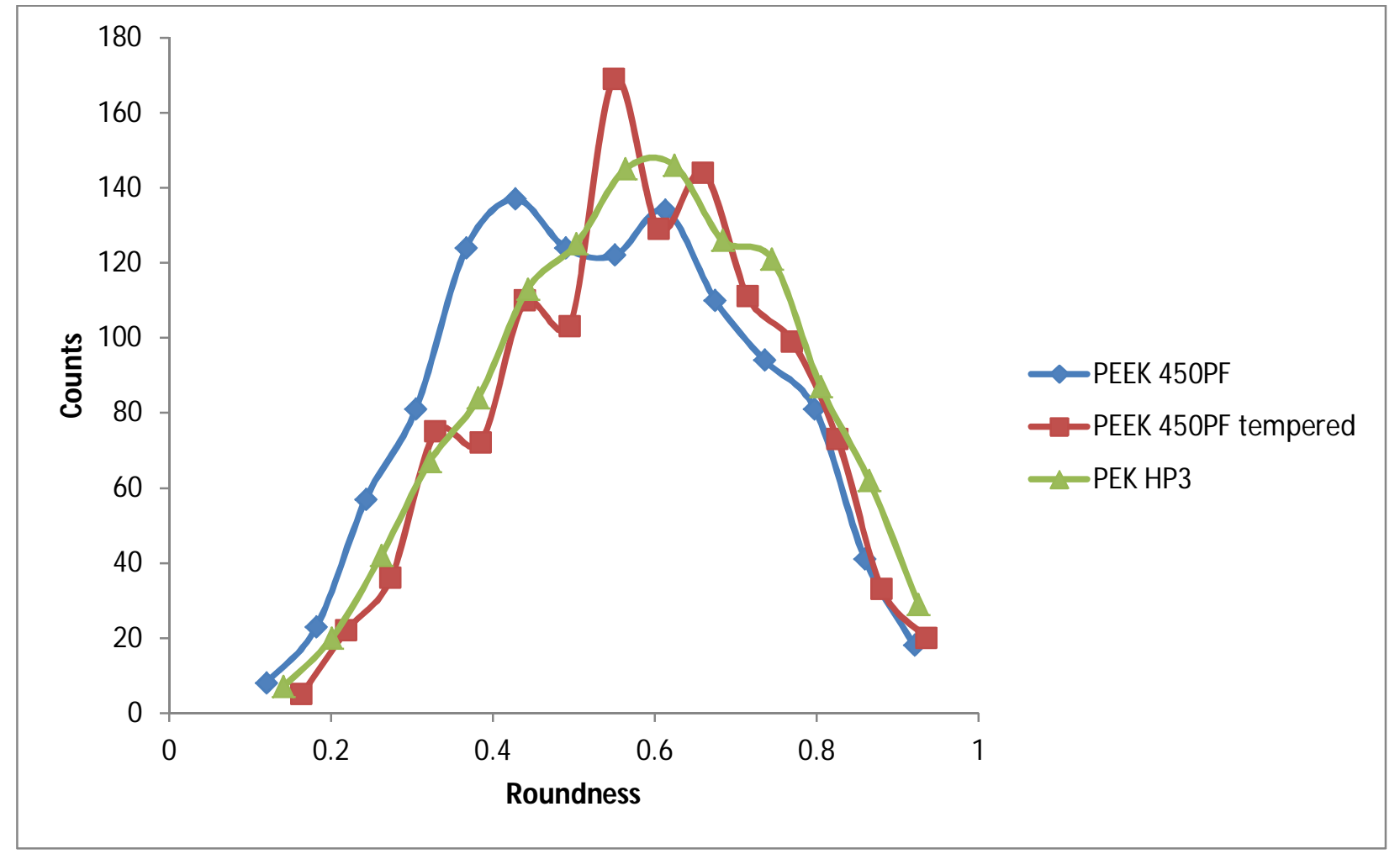

Figure 16. The shape descriptor Roundness in PEK HP3, virgin and thermally treated PEEK 450 PF

Figure 16 shows improved roundness of the tempered particles with a value distribution closer to that of the PEK HP3 particles. The influence of the powder particle shape on the layer spreading performance and formation of a good quality powder bed were investigated through the spreading tests carried out in the EOSINT P 800 system. Ten layers of fresh powders were spread across the building chamber at room temperature and visually inspected. Figure 17 shows the results of PEK HP3 (A), virgin PEEK 450PF (B) and tempered PEEK 450PF.
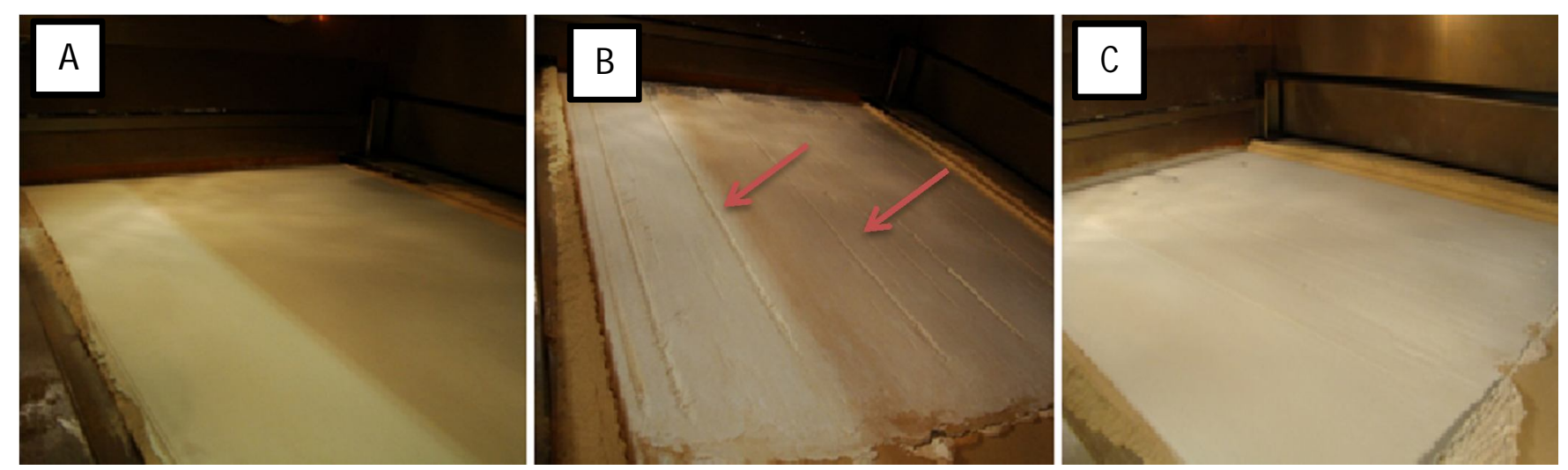

Figure 17. Spreading of (A) PEK HP3, virgin PEEK 450PF (B) and PEEK 450PF tempered (C) within the P 800 system 
In Figure $17 \mathrm{~B}$ there are clear marks of uneven layer deposition leading to non-uniform powder bed density. The shape of the powder particles in use can then strongly influence the formation of voids and uneven layers in the powder bed, causing cavities through successive layers of a laser sintered component.

The fracture surfaces of the ten layer samples of PEEK produced with different post sintering times (Figure 18 to Figure 21) and PEK HP3 (Figure 22) were also analysed with SEM in order to investigate the layer bonding properties across the cross-section of the samples.
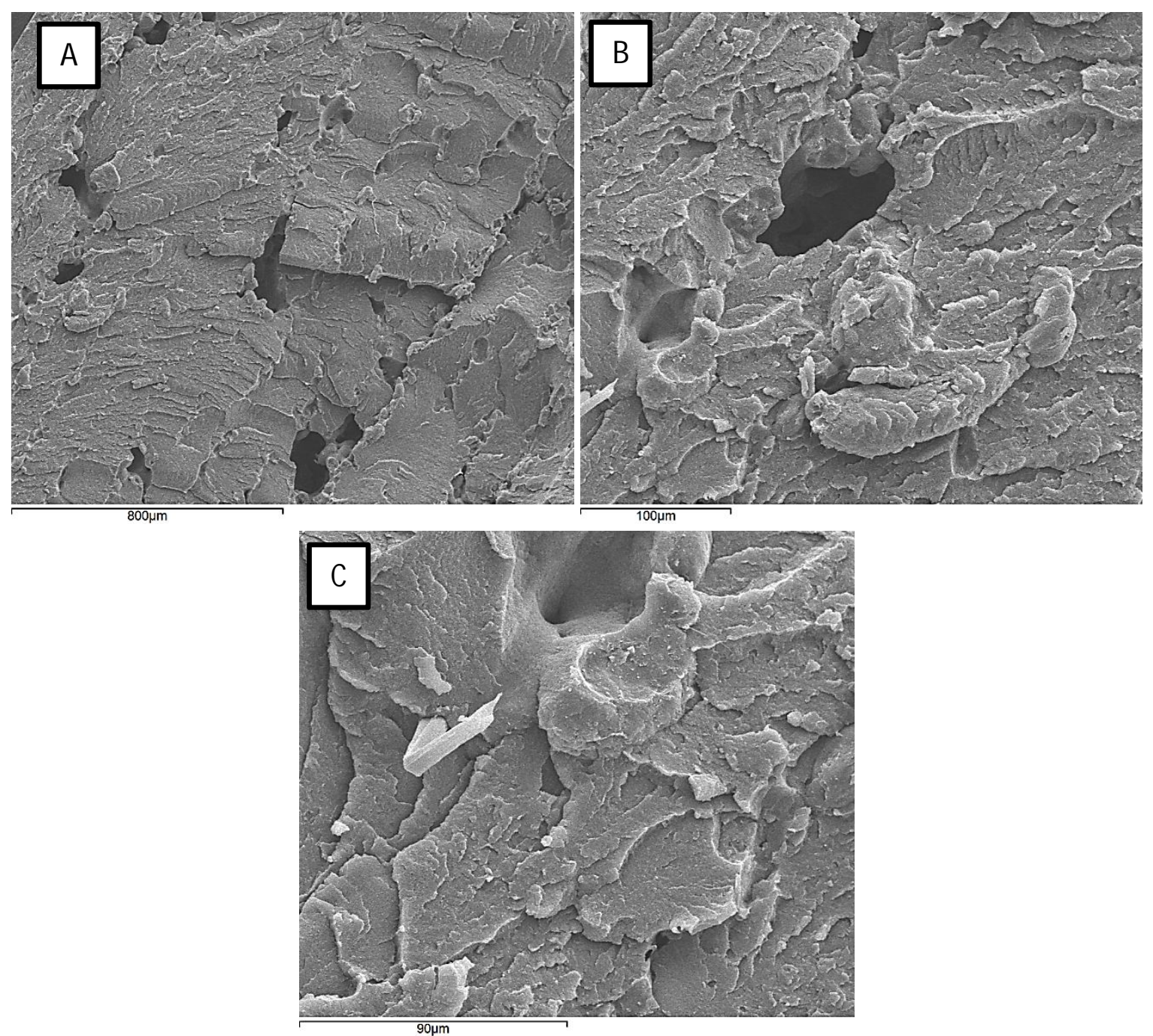

Figure 18. Fracture surface of PEEK ten layer specimen, $P S=6 \mathrm{~s}$, varied orders of magnification 

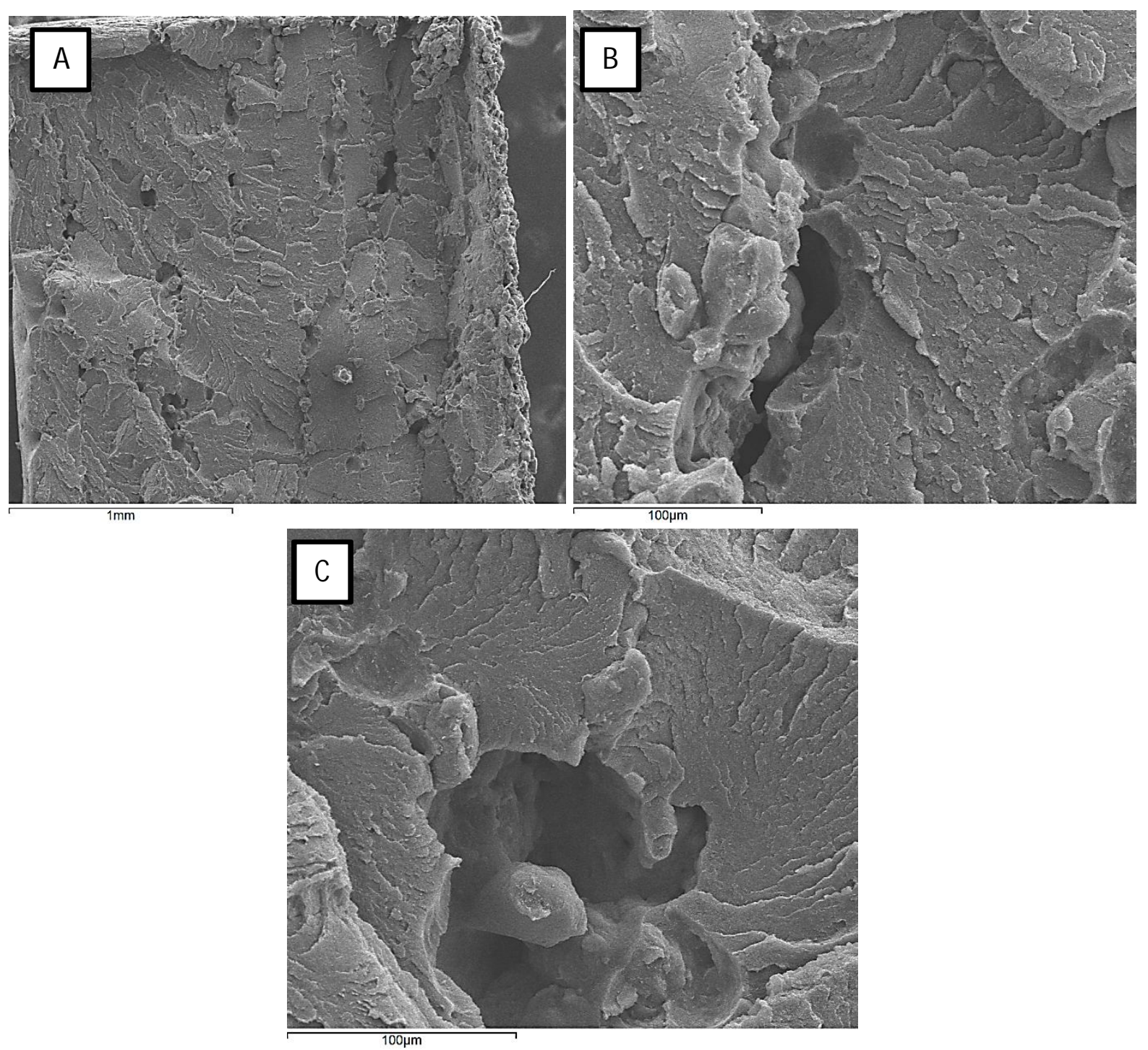

Figure 19. Fracture surface of PEEK ten layer specimen, $P S=9 \mathrm{~S}$, varied orders of magnification 

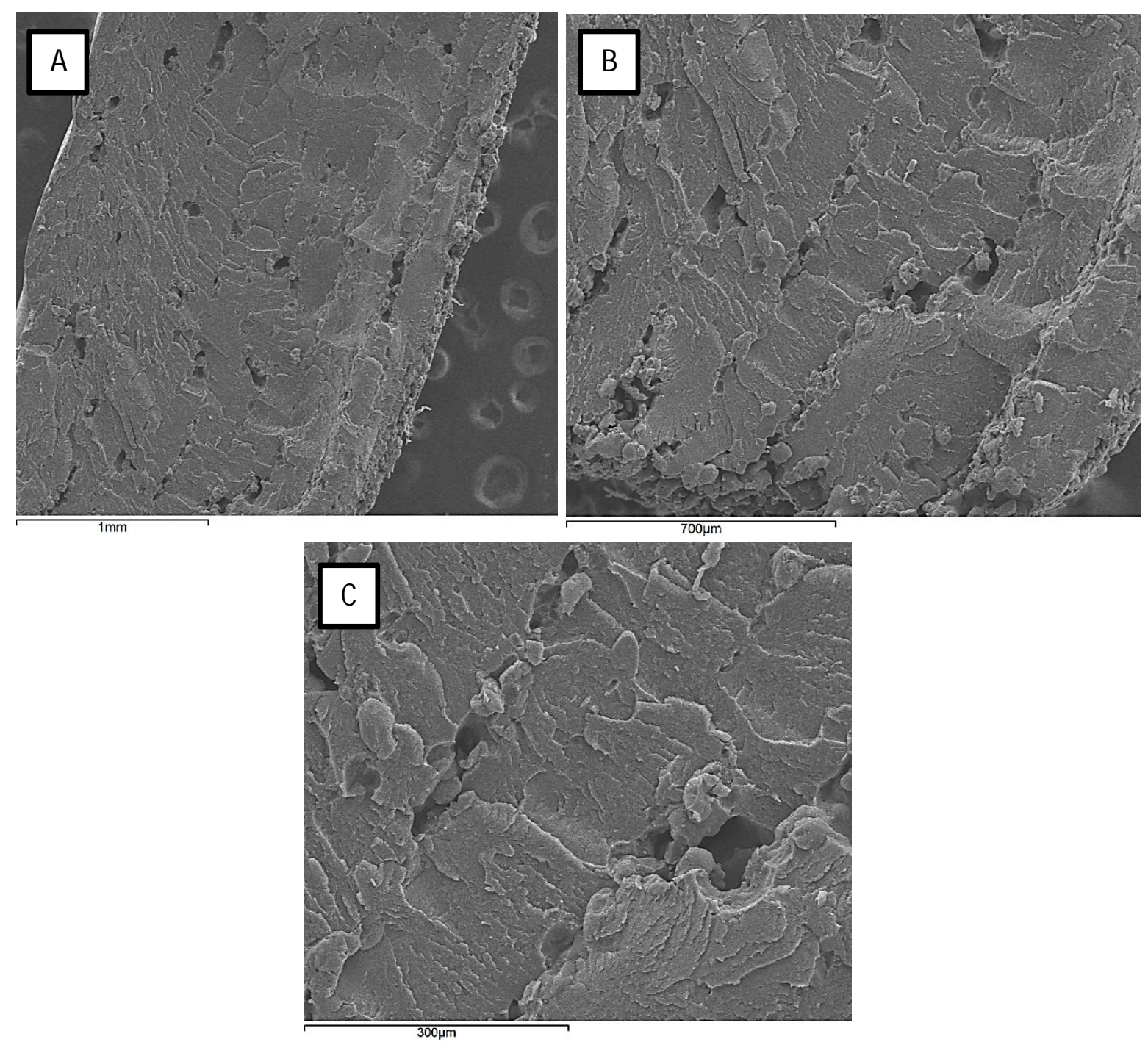

Figure 20. Fracture surface of PEEK ten layer specimen, $P S=12 \mathrm{~s}$, varied orders of magnification 


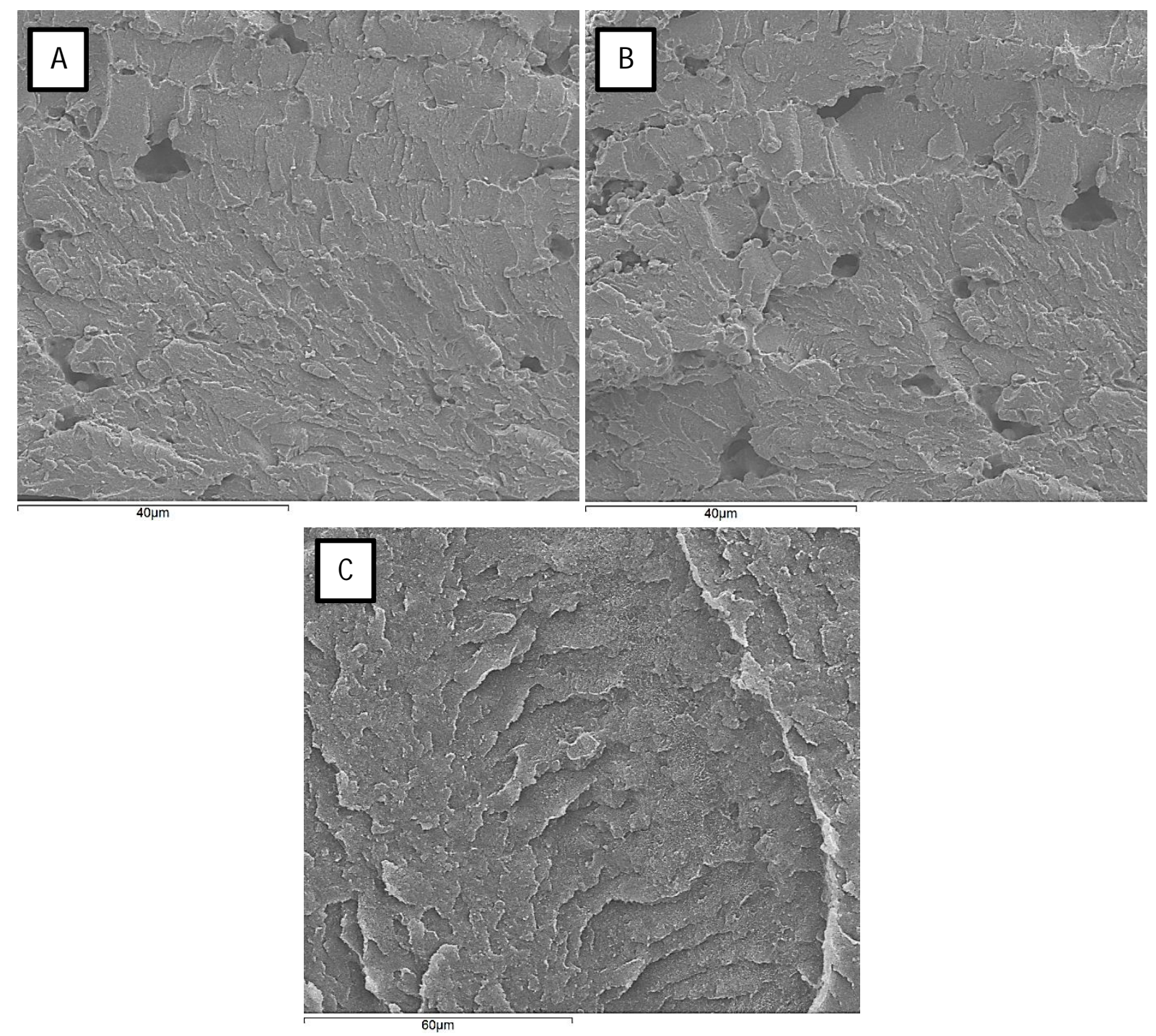

Figure 21. Fracture surface of PEEK ten layer specimen, $P S=15 \mathrm{~s}$, varied orders of magnification 

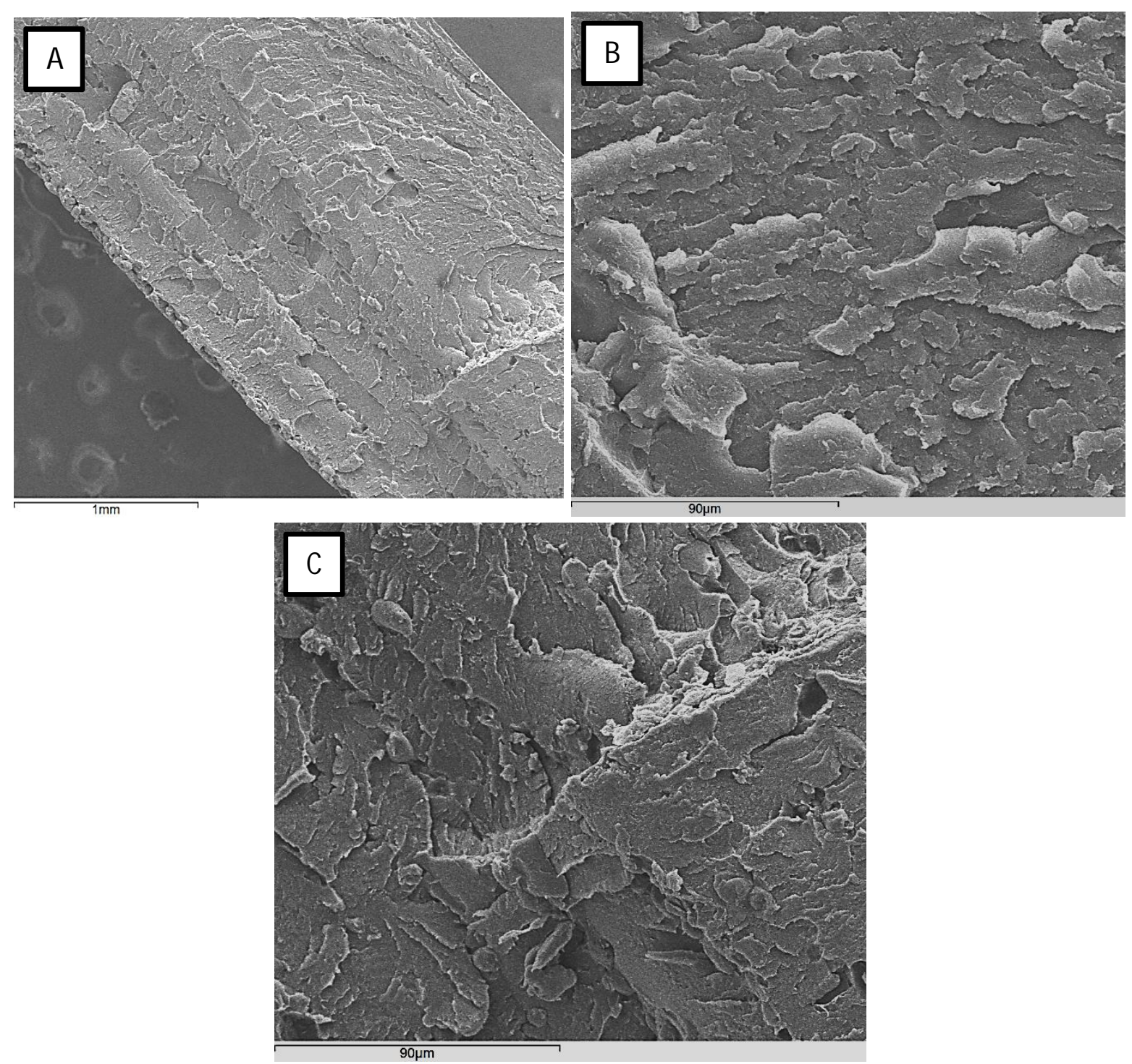

Figure 22. Fracture surface of PEK HP3 ten layer specimen (benchmark), PS $=12 \mathrm{~s}$, varied orders of magnification

All samples exhbited the layer structure typical of the laser sintering manufacturing process. While the PEEK samples with post sintering time of $6 s$ (Figure 18) and $9 \mathrm{~s}$ (Figure 19) showed large cavities, the samples with higher post sintering showed better bonding properties between the layers (Figure 20 and Figure 21). Interestingly, also the benchmark sample manufactured using PEK HP3 showed the presence of small cavities across the layers although of smaller size and smaller depth when compared to those of PEEK. This result helps to understand the differences found in the tensile properties between LS PEEK and PEK HP3 samples. 


\subsubsection{Surface morphology measurements profiles}

The one and ten layer specimens manufactured in PEEK material with post sintering times of $6 \mathrm{~s}, 9 \mathrm{~s}, 12 \mathrm{~s}$ and $15 \mathrm{~s}$ were also examined by means of the surface roughness parameter $\mathrm{Sa}$ [35] and compared to one and ten layer samples of PEK HP3 produced with post sintering time of $12 \mathrm{~s}$ as a benchmark.

For clarity only the PEEK samples built with post sintering time of $6 \mathrm{~s}$ (Figure 23 and Figure 24) and $12 \mathrm{~s}$ (Figure 25 and Figure 26) are shown. Figure 27 and Figure 28 exhibit the results obtained for PEK HP3 samples.

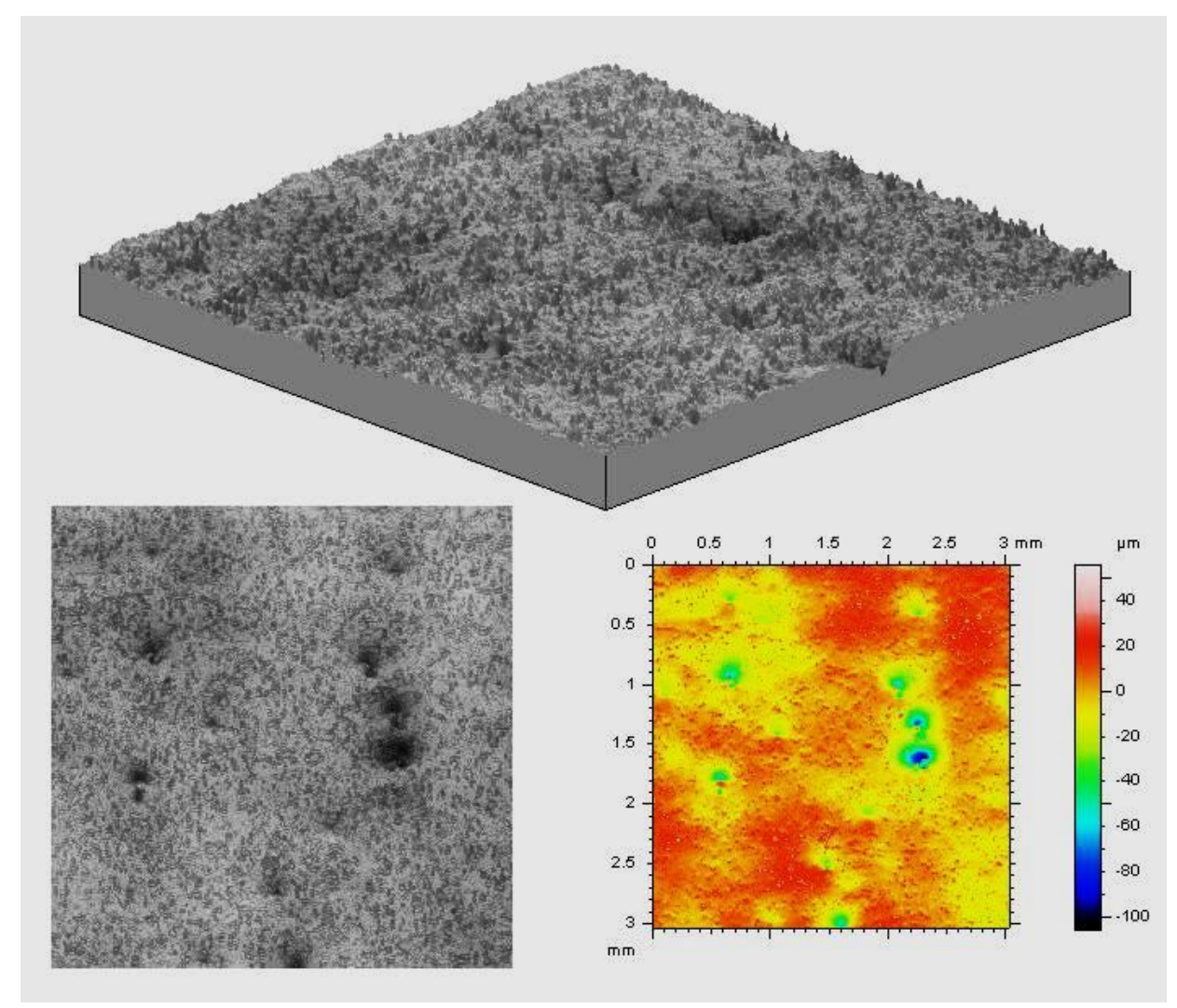

Figure 23. PEEK one layer specimen, $\mathrm{PS}=6 \mathrm{~s}$ 


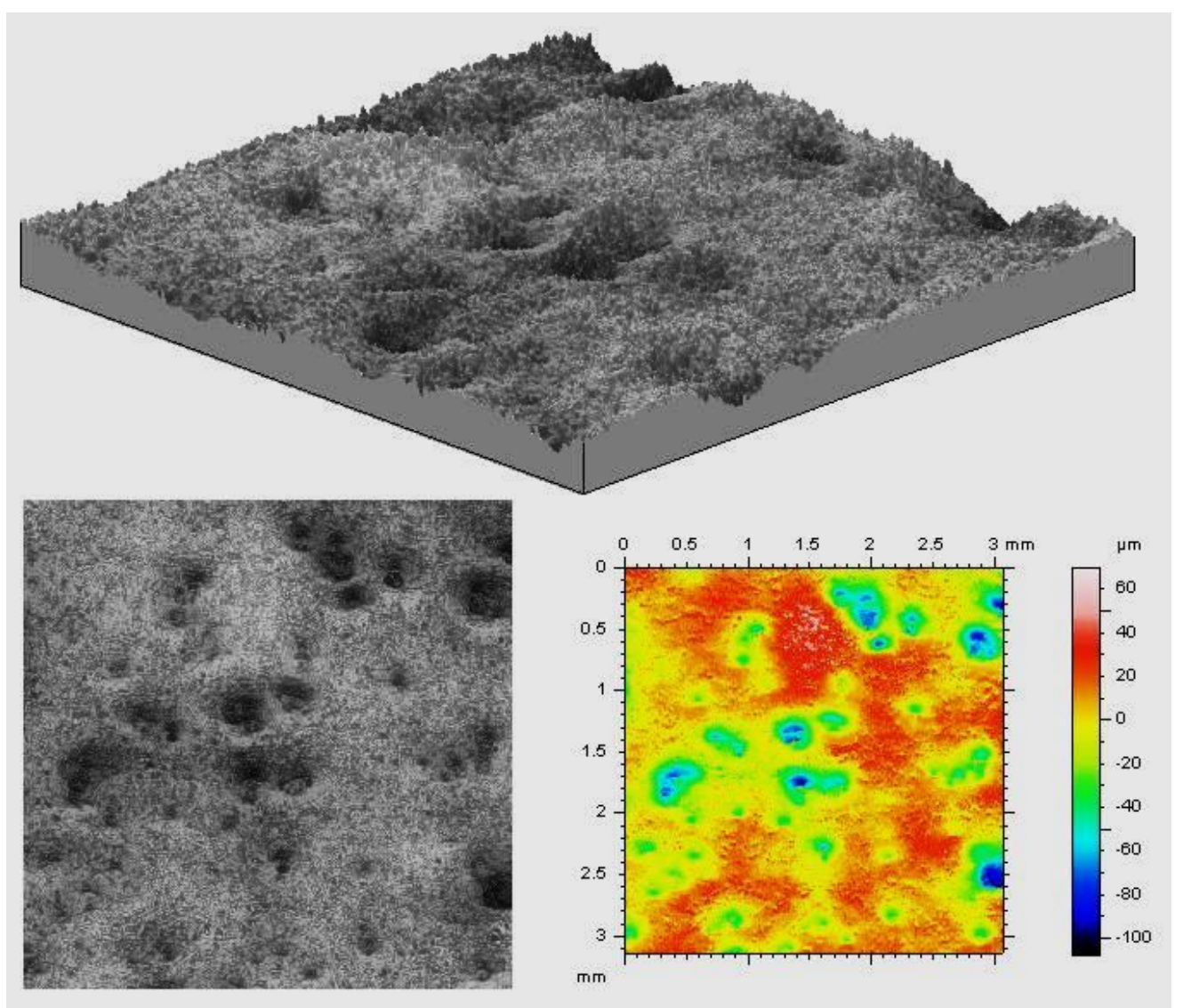

Figure 24. PEEK ten layer specimen, $P S=6 \mathrm{~s}$

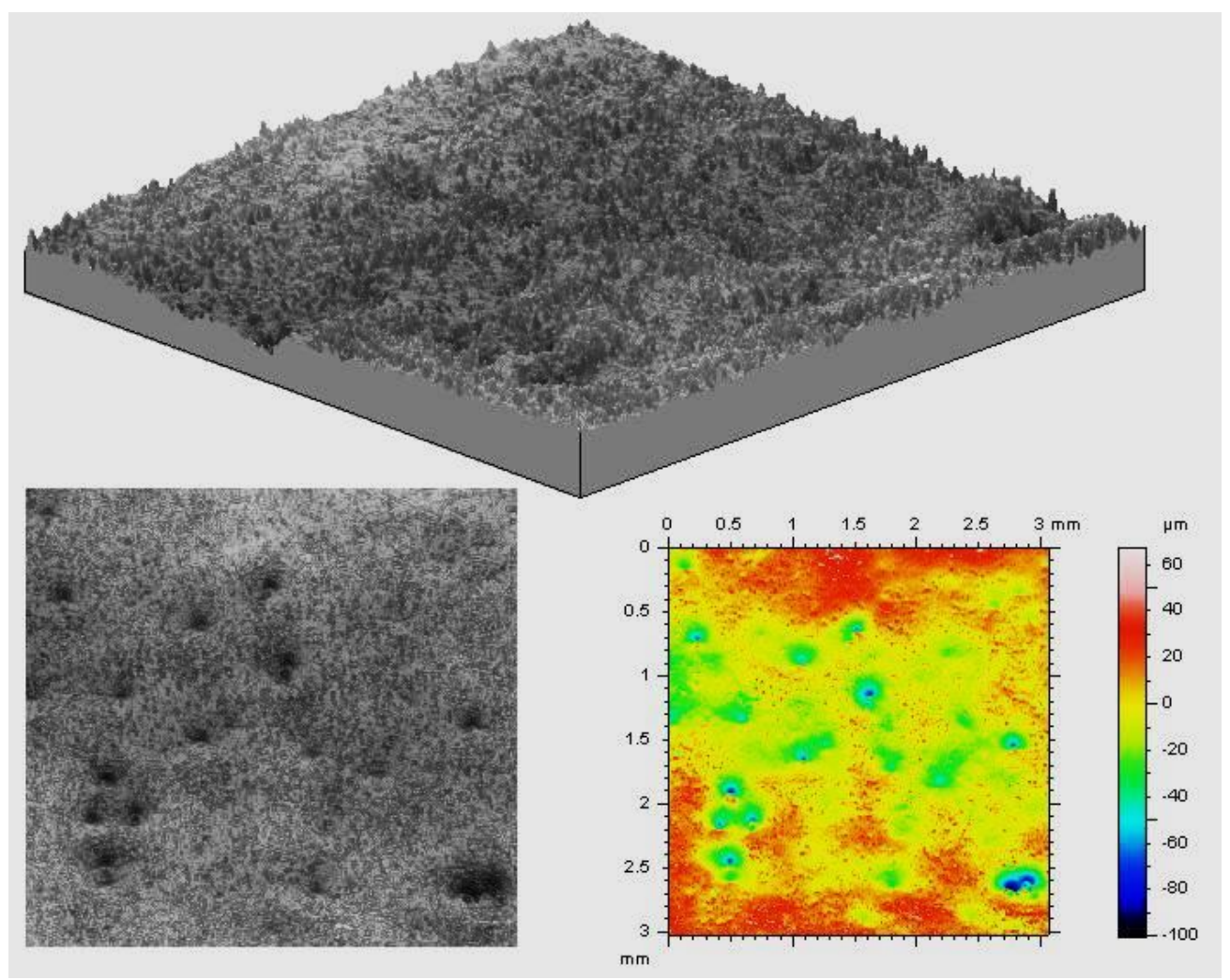

Figure 25. PEEK one layer specimen, $P S=12 \mathrm{~s}$ 


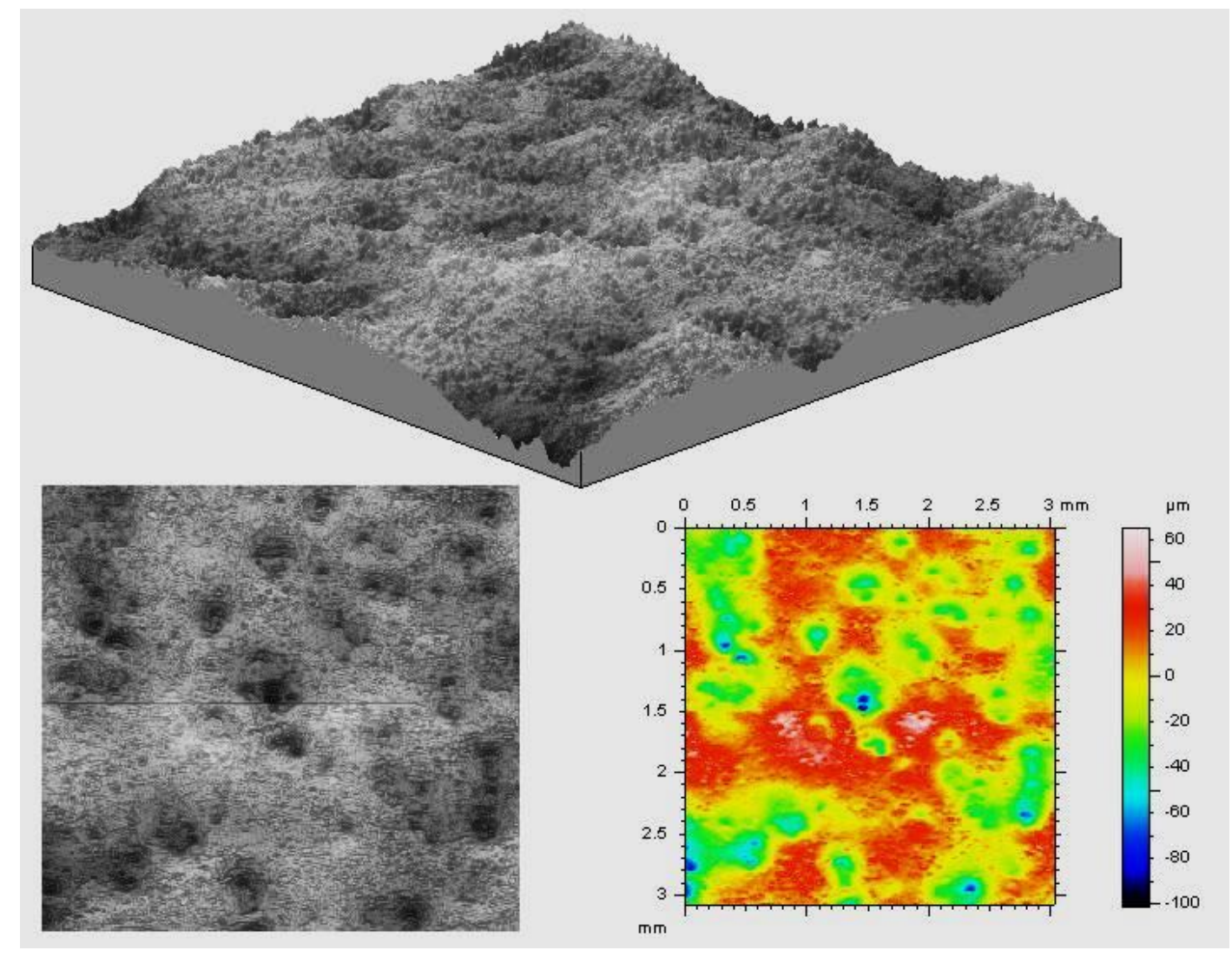

Figure 26. PEEK ten layer specimen, $P S=12 \mathrm{~s}$ 


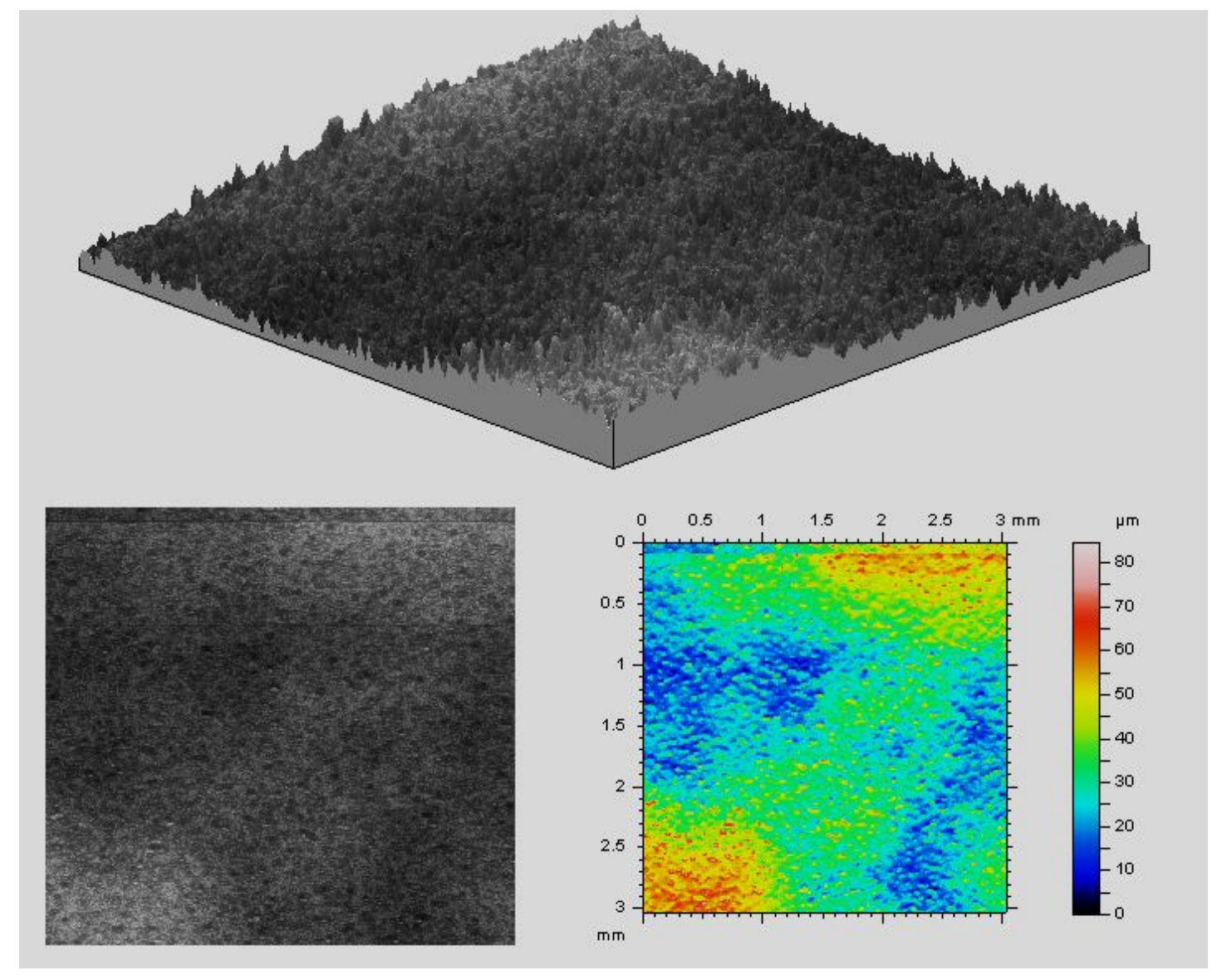

Figure 27. PEK HP3 one layer specimen, $P S=12 \mathrm{~s}$ 


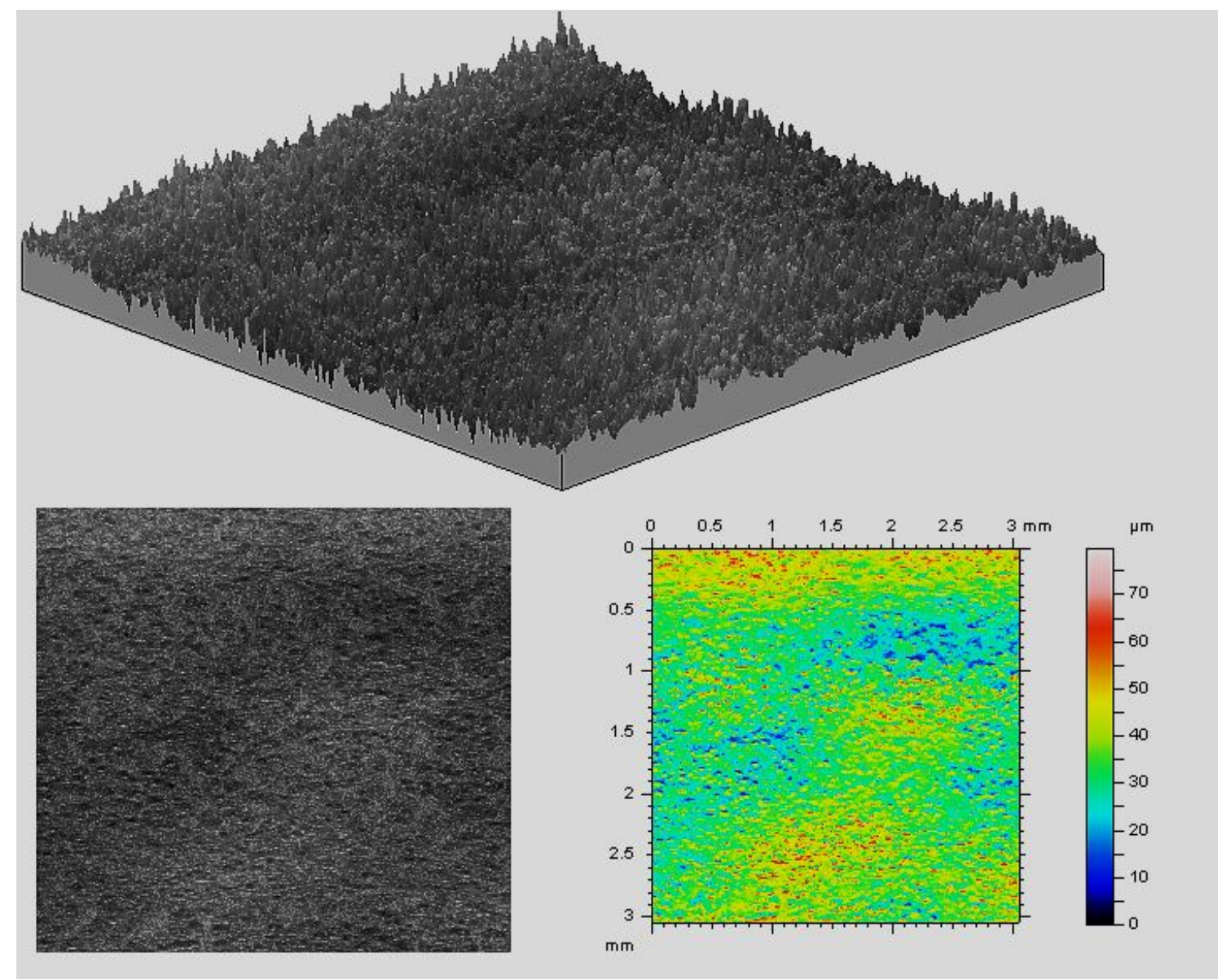

Figure 28. PEK HP3 ten layer specimens, PS $=12 \mathrm{~s}$

For all samples, surface roughness profiles outline the presence of cavities in the one and ten layer specimens as previously noticed with the SEM imaging. The numerical results of all samples are reported in Table 4 and Table 5.

\begin{tabular}{|l|l|}
\hline Sample -One layer & Sa $[\mu \mathrm{m}]$ \\
\hline PEEK 450 PF - 6 s & 8.7 \\
\hline PEEK 450 PF - 9 s & 9.27 \\
\hline PEEK 450 PF - 12 s & 12.8 \\
\hline PEEK 450 PF - 15 s & 10.9 \\
\hline Benchmark: PEK HP3 - 12s & 9.24 \\
\hline
\end{tabular}

Table 4. Surface roughness values of one layer samples

\begin{tabular}{|l|l|}
\hline Sample -Ten layer & Sa $[\mu \mathrm{m}]$ \\
\hline PEEK 450 PF -6 s & 15.1 \\
\hline
\end{tabular}




\begin{tabular}{|l|l|}
\hline PEEK 450 PF - 9 s & 16.9 \\
\hline PEEK 450 PF - 12 s & 16.5 \\
\hline PEEK 450 PF - 15 s & 19.4 \\
\hline Benchmark : PEK HP3 - 12 s & 6.9 \\
\hline
\end{tabular}

Table 5. Surface roughness values of ten layer samples

The surface roughness of the one layer specimens seems to increase with the post sintering time up to $12 \mathrm{~s}$ where it levels off. A possible explanation could be that a longer heating phase makes the viscosity of the just melted polymeric film decrease which can then flow easier amongst the particles creating the observed cavities. This mechanism enhances the irregularities of the powder packing instead of creating a smoother surface. When comparing the results of the PEEK one layer samples to PEK HP3 it can be noted that the PEEK sample with post sintering 9 s seems to better match the HP3 surface morphology.

It can also be noticed that ten layer specimens are rougher than one layer specimens for the PEEK samples. This could be explained with the same mechanism described above where the viscosity of a just sintered layer is lower than the viscosity of the layers underneath and therefore the polymer can easily flow within the irregularities of the below powder arrangements and creates large craters. The ten layer sample of PEK HP3 manufactured with post sintering time of 12 seconds showed a significant decrease in the surface roughness of the samples, with only small cavities and irregularities. As suggested in previous studies [36] this result could be the consequence of two simultaneous contributions: the particle morphology and melt viscosity. As shown in Figure 17 and previous studies [22], PEK HP3 shows higher values of shape descriptors such as roundness, circularity, aspect ratio and solidity compared to PEEK, leading to higher flowability, therefore higher powder bed density after the layer spreading and ultimately smoother and denser ten layer samples. In addition, PEEK 450PF and HP3 PEK have different melt viscosities: PEK is less viscous than PEEK 450PF, according to the datasheets of PEK and PEEK grades supplied by Victrex [9]. The lower viscosity of the PEK material combined with better flowability helps the formation of a more homogenous and smooth sintered layers than in PEEK.

Figure 29 shows some examples of profiles extracted from the following PEEK samples: (A) one layer specimen with PS $=6 \mathrm{~s}$; (B) ten layer specimen with PS $=6 \mathrm{~s}$; (C) ten layer specimen with $P S=12 \mathrm{~s}$ and (D) ten layer specimen with $P S=15 \mathrm{~s}$. Sample $E$ is the ten layer HP3 PEK sample. In sample A the maximum variation is around $100 \mu \mathrm{m}$, while in sample $B$ it is around $120 \mu \mathrm{m}$, value equal to the layer thickness set in the P 800 system for the manufacture of the samples. This shows how in the case of one layer specimens cavities are less deep than in ten layer specimens. A variation of $120 \mu \mathrm{m}$ also proves that the irregularities of the ten layer samples are through the last sintered layer of powder. Samples 
$C$ and $D$ have respectively maximum deviations of 140 and nearly $200 \mu \mathrm{m}$, indicating that with longer post sintering times cavities and irregularities occur through more than one layer. Lastly, as expected from the results in Table 5, the PEK HP3 sample (E) showed the smallest deviation, sign of a fairly smooth and regular surface.

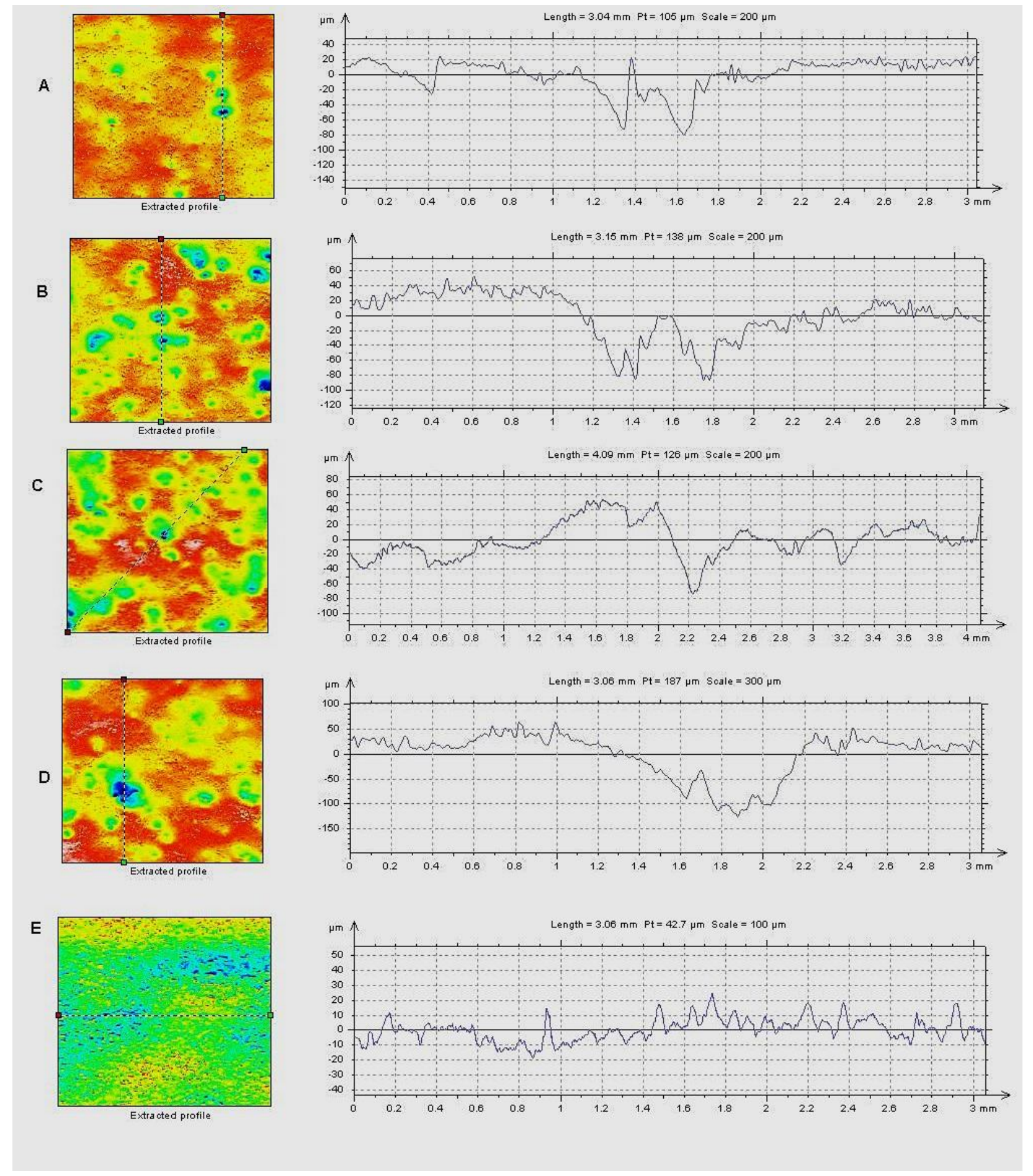

Figure 29. Example of cavities in the profile of one and ten layer specimens: (A) PEEK 450PF one layer, $P S=6 \mathrm{~S}$; (B) PEEK 450PF ten layer, PS = 6 s; (C) PEEK 450PF ten layer, PS =12 s; (D) PEEK 450PF ten layer, PS =15 s; (E) PEK HP3 ten layer, PS $=12 \mathrm{~s}$. 


\subsubsection{Tensile testing results}

The performance of the tensile samples built according to build 5 with different post sintering times, are shown in Figure 30.

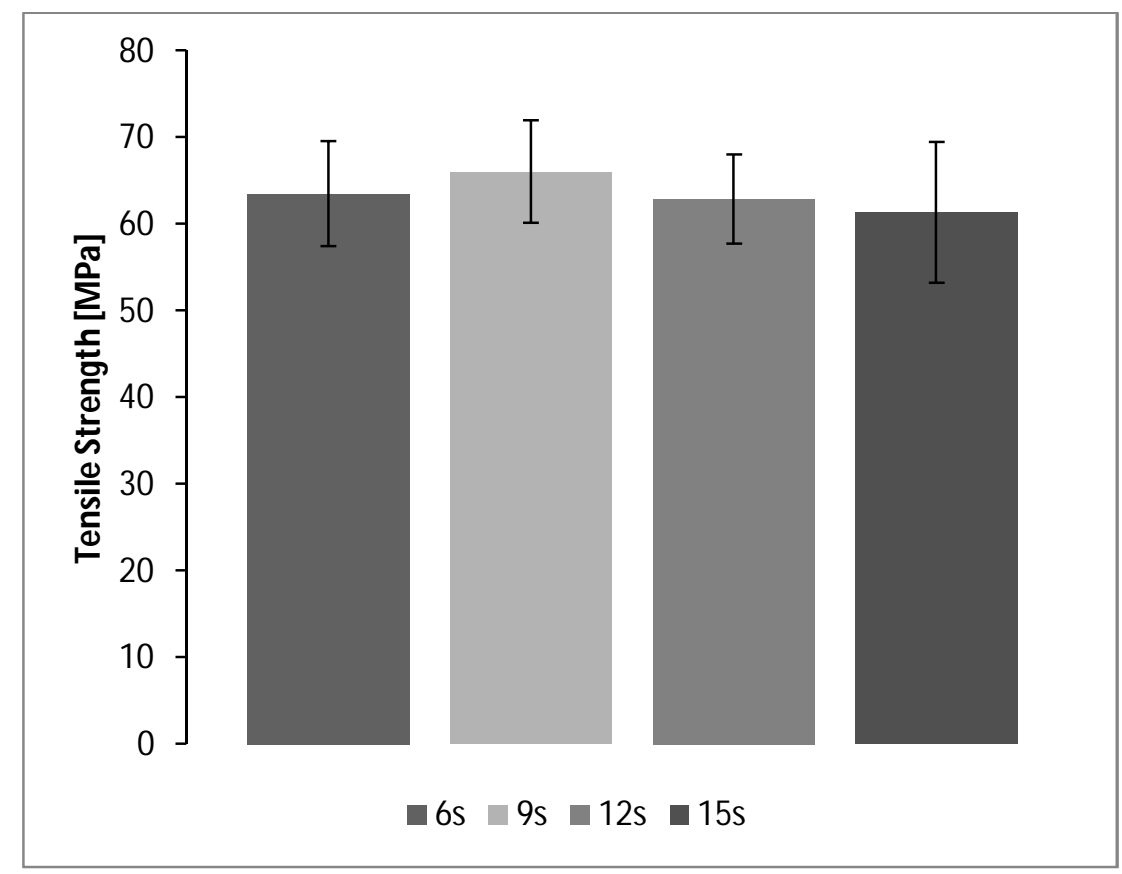

Figure 30. Tensile strength values and standard deviation bars of build 5 samples with different post sintering times

The tensile strength values of the four groups seem not affected by the duration of the post sintering in the range $6-15 \mathrm{~s}$. It can be then pointed out that decreasing the post sintering up to $6 \mathrm{~s}$ will not significantly affect the tensile strength of the final products. 


\subsubsection{DMA results}

Figure 31 and Figure 32 show the storage and loss moduli for the samples built according to build 5 with post sintering times of $6,9,12$ and $15 \mathrm{~s}$.

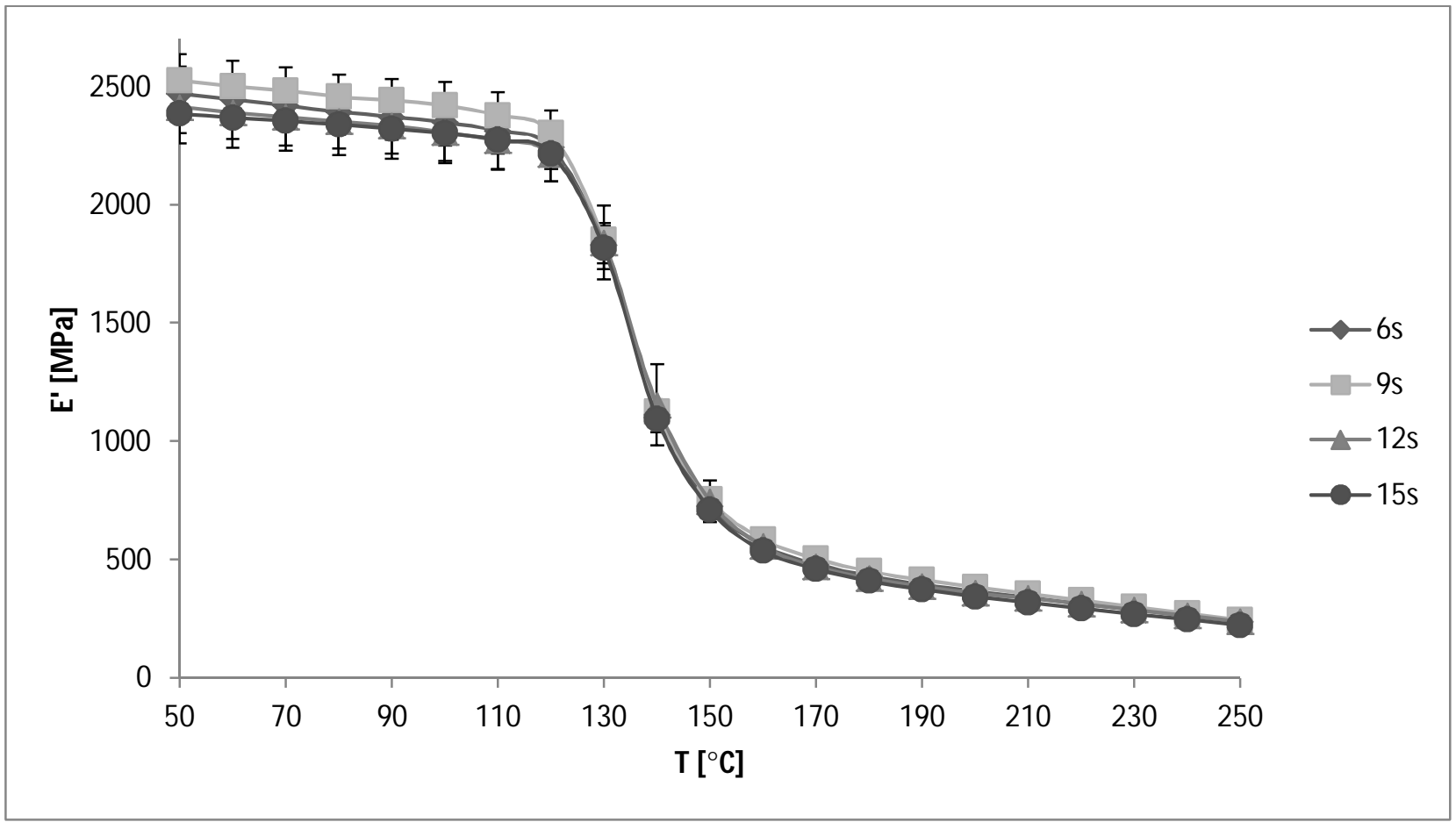

Figure 31. Storage Modulus E' for different PS times

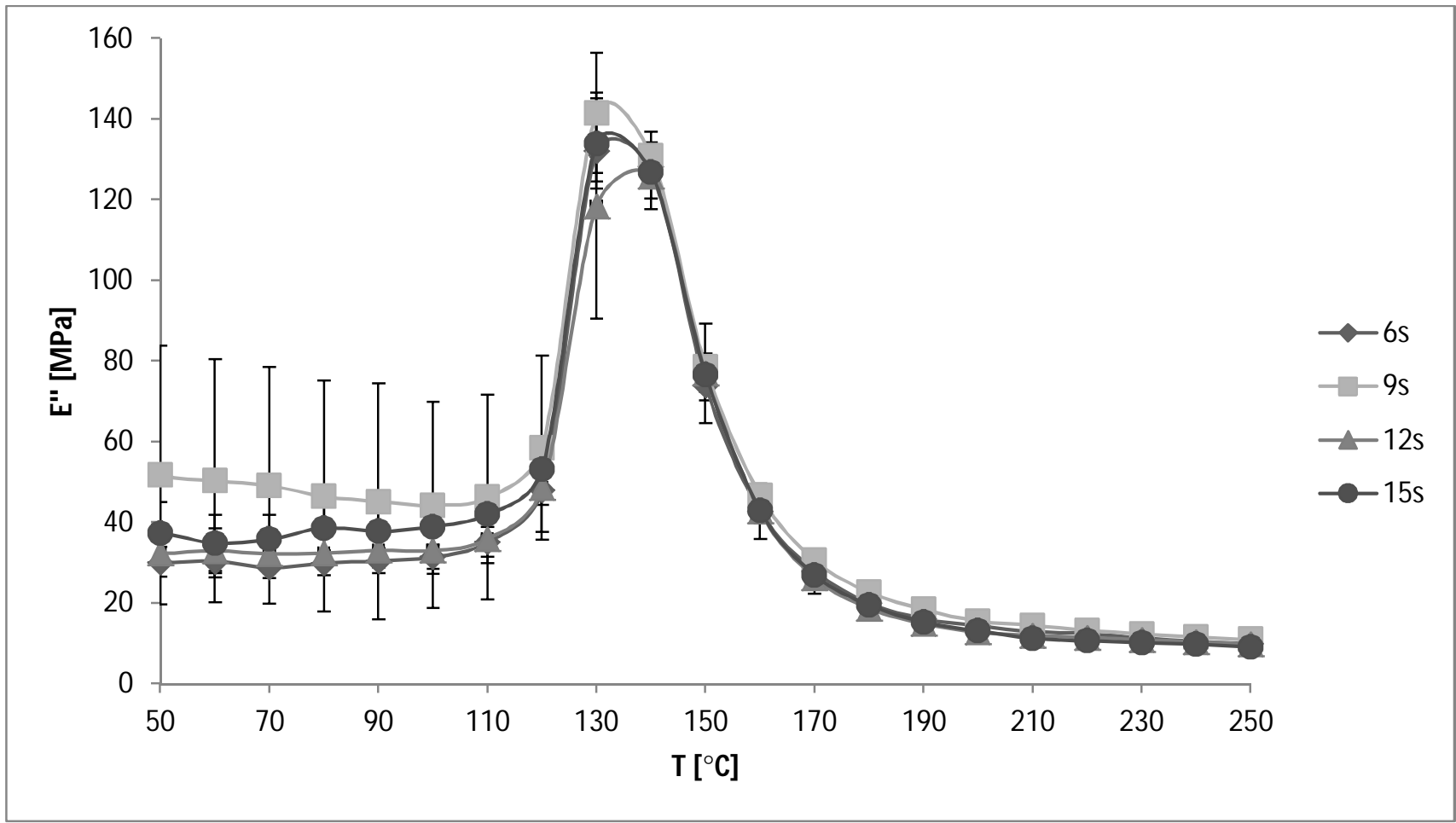

Figure 32. Loss M odulus E" for varied PS times 
From the Figure 31 and Figure 32, it appears clear that both storage and loss moduli are not affected by the post sintering time, result also found in the tensile testing characterisation.

\section{Conclusions}

This study is the first on high temperature laser sintering of PEEK material. Following an optimisation process, the tensile strength of the PEEK components achieved a storage modulus of $2500 \mathrm{M} \mathrm{Pa}$ and a tensile strength value of $63 \mathrm{M} \mathrm{Pa}$, only $20 \mathrm{M} \mathrm{Pa}$ lower than the current commercial grade HP3 PEK. The post sintering, a feature characteristic unique to the high temperature laser sintering process, does not seem to be sensitive to the exposure time in the case of PEEK. The mechanical properties in the range $6-15 \mathrm{~s}$ post sintering times are similar. The particle morphology and particle flow play an important part on the manufacturing process, and link with the post-sintering profiles. The experimental results of this work can then open the way to the use of PEEK in laser sintering for the manufacture of high performance engineering components.

\section{Acknowledgements}

The authors wish to acknowledge Victrex [9] for the supply of materials and especially Andy Anderson for the technical assistance during the preparation of the paper. Special thanks go to the engineering specialists Richard Davies and J ames Bradbury for their expertise and help. The support of Dr Davood Rouholamin in the $\mu$ CT scanning and Dr Yuan Wang in the DM A of PEK components is also acknowledged.

\section{References}

1. Wohlers, T.T. and W. Associates, Wohlers Report 2012: Additive M anufacturing and Three Dimensional Printing State of the Industry Annual2012: Wohlers Associates.

2. Kurtz, S.M . and J.N. Devine, PEEK biomaterials in trauma, orthopedic, and spinal implants. Biomaterials, 2007. 28(32): p. 4845-4869.

3. EOS, http://www.eos.info/en/home.html.

4. Ghita, O.R., et al., Physico-chemical behaviour of Poly (Ether Ketone) (PEK) in High Temperature Laser Sintering (HT-LS). Journal of M aterials Processing Technology, 2013.

5. EOS. EOS HP3. Available from: http://eos.materialdatacenter.com/eo/material/pdf/datasheet/? mdc5 $=57$ l4t4ljequ5 h26ogk4m7d4s46.

6. Tan, K.H., et al., Scaffold development using selective laser sintering of polyetheretherketone-hydroxyapatite biocomposite blends. Biomaterials, 2003. 24(18): p. 3115-3123.

7. Tan, K.H., et al., Selective laser sintering of biocompatible polymers for applications in tissue engineering. Bio-M edical M aterials and Engineering, 2005. 15(1-2): p. 113-124. 
8. Tan, K.H., et al., Fabrication and characterization of three-dimensional poly (etherether-ketone) / hydroxyapatite biocomposite scaffolds using laser sintering. Proceedings of the Institution of M echanical Engineers, Part $\mathrm{H}$ : Journal of Engineering in M edicine, 2005. 219(3): p. 183-194.

9. Victrex. http://www. victrex.com.

10. 3DSystems, http://www.3dsystems.com/.

11. Rechtenwald T., E.G., Comparison between laser sintering of PEEK and PA using design of experiment methods. Virtual M odelling and Rapid M anufacturing Advanced Research in Virtual and Rapid Prototyping, 2005.

12. Pohle, D., et al., Processing of Three-Dimensional Laser Sintered Polyetheretherketone Composites and Testing of Osteoblast Proliferation in vitro. Macromolecular Symposia, 2007. 253(1): p. 65-70.

13. Evonik. http://corporate.evonik.com/en/Pages/default.aspx.

14. von Wilmowsky, C., et al., Effects of bioactive glass and B-TCP containing threedimensional laser sintered polyetheretherketone composites on osteoblasts in vitro. Journal of Biomedical M aterials Research Part A, 2008. 87A(4): p. 896-902.

15. Von Wilmonsky, C., et al., In Vivo Evaluation of ß-TCP Containing 3D Laser Sintered Poly(ether ether ketone) Composites in Pigs. Journal of Bioactive and Compatible Polymers, 2009. 24(2): p. 169-184.

16. Wegner, A. and G. Witt, Correlation of Process Parameters and Part Properties in Laser Sintering using Response Surface M odeling. Physics Procedia, 2012. 39(0): $p$. 480-490.

17. Woicke N, Wagner T., Eyerer P., Carbon Assisted Laser Sintering of Thermoplastic Polymers. ANTEC conference proceedings, 2005. 7: p. 36-40.

18. Woicke N., K.M., Eyerer P., Rapid Prototyping of PEEK Parts by the M eans of LaserSintering, 2004.

19. Kroh, M., C. Bonten, and P. Eyerer, Improvement of mechanical properties by additive assisted laser sintering of PEEK. AIP Conference Proceedings, 2014. 1593: $p$. 724-727.

20. Beard, M.A., et al., M aterial characterisation of Additive M anufacturing components made from a polyetherketone (PEK) high temperature thermoplastic polymer, in Innovative Developments in Virtual and Physical Prototyping2011, CRC Press. p. 329332.

21. Ghita, O., et al., High Temperature Laser Sintering (HT-LS): An investigation into mechanical properties and shrinkage characteristics of Poly (Ether Ketone) (PEK) structures. Materials \& Design, 2014. 61(0): p. 124-132.

22. Berretta, S., O. Ghita, and K.E. Evans, M orphology of polymeric powders in Laser Sintering (LS): From Polyamide to new PEEK powders. European Polymer Journal, 2014. 59: p. 218-229.

23. EOS, EOSINT P800 manual.

24. Gibson, I. and D.P. Shi, M aterial properties and fabrication parameters in selective laser sintering process. Rapid prototyping journal, 1997. 3(4): p. 129-136.

25. CTPro3D. http://www.nikonmetrology.com/en EU/News/US-News/NikonMetrology-incorporates-Inspect-X-and-CT-Pro-into-XT-software-Suite-V2.2.

26. VGStudioM ax2.1. http://vgstudio-max.software.informer.com/2.1/.

27. SPSS, I. http://www-01.ibm.com/software/analytics/spss/. 
28. Soe, S.P., Quantitative analysis on SLS part curling using EOS P700 machine. Journal of M aterials Processing Technology, 2012. 212(11): p. 2433-2442.

29. Caulfield, B., P.E. M cHugh, and S. Lohfeld, Dependence of mechanical properties of polyamide components on build parameters in the SLS process. Journal of M aterials Processing Technology, 2007. 182(1-3): p. 477-488.

30. Vasquez, M., B. Haworth, and N. Hopkinson, M ethods for quantifying the stable sintering region in laser sintered polyamide-12. Polymer Engineering \& Science, 2013. 53(6): p. 1230-1240.

31. Thomas, S. and V.P. M, Handbook of Engineering and Specialty Thermoplastics: Volume 3: Polyethers and Polyesters2011: Wiley.

32. Collyer, A.A., A Practical Guide to the Selection of High-Temperature Engineering Thermoplastics2013: Elsevier Science.

33. Ho, H.C.H., I. Gibson, and W.L. Cheung, Effects of energy density on morphology and properties of selective laser sintered polycarbonate. Journal of Materials Processing Technology, 1999. 89-90(0): p. 204-210.

34. NHS. Image J. Available from: http://rsbweb.nih.gov/ij/.

35. Mainsah, E., J.A. Greenwood, and D. Chetwynd, M etrology and Properties of Engineering Surfaces2001: Springer.

36. Goodridge, R.D., C.J. Tuck, and R.J.M. Hague, Laser sintering of polyamides and other polymers. Progress in M aterials Science, 2012. 57(2): p. 229-267. 